\title{
Targeting cancer metabolism by simultaneously disrupting parallel nutrient access pathways
}

\author{
Seong M. Kim, ${ }^{1}$ Saurabh G. Roy, ${ }^{1}$ Bin Chen, ${ }^{2}$ Tiffany M. Nguyen, ${ }^{1}$ Ryan J. McMonigle, ${ }^{1}$ Alison N. McCracken, ${ }^{1}$ Yanling Zhang, ${ }^{3}$ \\ Satoshi Kofuji, ${ }^{4}$ Jue Hou, ${ }^{5}$ Elizabeth Selwan, ${ }^{1}$ Brendan T. Finicle, ${ }^{1}$ Tricia T. Nguyen, ${ }^{1}$ Archna Ravi, ${ }^{1}$ Manuel U. Ramirez, ${ }^{1}$ Tim Wiher, ${ }^{1}$ \\ Garret G. Guenther, ${ }^{1}$ Mari Kono, ${ }^{6}$ Atsuo T. Sasaki, ${ }^{4}$ Lois S. Weisman, ${ }^{3}$ Eric O. Potma, ${ }^{5}$ Bruce J. Tromberg, ${ }^{5}$ Robert A. Edwards, ${ }^{7}$ \\ Stephen Hanessian, ${ }^{2,8}$ and Aimee L. Edinger ${ }^{1}$ \\ 'Department of Developmental and Cell Biology, University of California Irvine (UCI), Irvine, California, USA. ²Department of Chemistry, Université de Montréal, Montréal, Québec, Canada. ${ }^{3}$ Department of Cell and \\ Developmental Biology, University of Michigan, Ann Arbor, Michigan, USA. Departments of Internal Medicine, Neurosurgery, and Cancer Biology, University of Cincinnati College of Medicine, Cincinnati, Ohio, USA. \\ ${ }^{5}$ Department of Biomedical Engineering, UCI, Irvine, California, USA. ${ }^{6}$ National Institute of Diabetes and Digestive and Kidney Diseases (NIDDK), Bethesda, Maryland, USA. 'Department of Pathology, \\ University of California Irvine School of Medicine, Irvine, California, USA. ${ }^{8}$ Department of Pharmaceutical Sciences, UCI, Irvine, California, USA.
}

\begin{abstract}
Oncogenic mutations drive anabolic metabolism, creating a dependency on nutrient influx through transporters, receptors, and macropinocytosis. While sphingolipids suppress tumor growth by downregulating nutrient transporters, macropinocytosis and autophagy still provide cancer cells with fuel. Therapeutics that simultaneously disrupt these parallel nutrient access pathways have potential as powerful starvation agents. Here, we describe a water-soluble, orally bioavailable synthetic sphingolipid, SH-BC-893, that triggers nutrient transporter internalization and also blocks lysosome-dependent nutrient generation pathways. SH-BC-893 activated protein phosphatase 2A (PP2A), leading to mislocalization of the lipid kinase PIKfyve. The concomitant mislocalization of the PIKfyve product PI(3,5)P2 triggered cytosolic vacuolation and blocked Iysosomal fusion reactions essential for LDL, autophagosome, and macropinosome degradation. By simultaneously limiting access to both extracellular and intracellular nutrients, SH-BC-893 selectively killed cells expressing an activated form of the anabolic oncogene Ras in vitro and in vivo. However, slower-growing, autochthonous PTEN-deficient prostate tumors that did not exhibit a classic Warburg phenotype were equally sensitive. Remarkably, normal proliferative tissues were unaffected by doses of SH-BC-893 that profoundly inhibited tumor growth. These studies demonstrate that simultaneously blocking parallel nutrient access pathways with sphingolipid-based drugs is broadly effective and cancer selective, suggesting a potential strategy for overcoming the resistance conferred by tumor heterogeneity.
\end{abstract}

\section{Introduction}

To meet the anabolic demands of cell division, oncogenic mutations drive glucose and glutamine transporter gene expression $(1-4)$. The LDL receptor is similarly upregulated in cancer cells to provide exogenous cholesterol and fatty acids that fuel cell growth $(5,6)$. Oncogenic signaling pathways also promote nutrient uptake posttranscriptionally by preventing the lysosomal degradation of these nutrient transport proteins (7). Tumors with activated Ras acquire additional extracellular nutrients via macropinocytosis, an endocytic process that produces amino acids when engulfed proteins are degraded in the lysosome $(8,9)$. Cancer cells are "addicted" to these nutrient influx pathways, because oncogenic mutations create a continuous, high demand for fuel and limit metabolic flexibility. A classic example of how this addiction can be exploited therapeutically is the use of L-asparaginase to kill acute lymphoblastic leukemia cells that cannot synthesize sufficient quantities of the nonessential amino acid asparagine to meet their metabolic demand (10). Preclinical studies show that a subset of human cancers likewise requires imported LDL, arginine, serine, or glycine

Conflict of interest: The authors have declared that no conflict of interest exists. Submitted: February 18, 2016; Accepted: August 16, 2016.

Reference information: J Clin Invest. 2016;126(11):4088-4102. doi:10.1172/JCI87148. for growth and survival $(5,11-13)$. These studies demonstrate that limiting nutrient uptake can selectively eliminate transformed cells and also highlight that the specific nutrient addictions of different cancer classes diverge depending on the molecular defects present.

An increasingly sophisticated understanding of how individual oncogenes and tumor suppressors alter flux through key metabolic pathways and the expanding ability to catalog the mutations present in tumors will facilitate the use of targeted metabolic therapies. However, tumor heterogeneity limits the effectiveness of these agents. Preexisting tumor cells that rely on a distinct set of anabolic enzymes would be enriched during treatment with small-molecule metabolic inhibitors, thereby contributing to the development of resistance $(14,15)$. Selective pressures may also promote rewiring of metabolic pathways in tumor cells that are crippled but not killed by targeted metabolic therapies, akin to what has been observed with cytostatic agents targeting oncogenic signal transduction pathways (16). One means to circumvent these hurdles would be to target the apex of the anabolic pyramid, that of nutrient uptake. No matter which biosynthetic pathways are essential in a given tumor cell, exogenous nutrients will be required to build biomass. If access to multiple nutrients could be restricted simultaneously, many different tumor classes would be sensitive and potential resistance pathways suppressed. 
A<smiles>[R20]CCCCCCc1ccc(CCC(N)(CO)CO)cc1</smiles><smiles>CCCCCCCc1ccc([C@@H]2CCNC2CO)cc1</smiles>

B

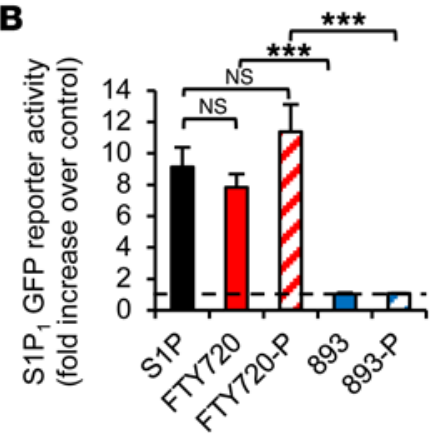

C

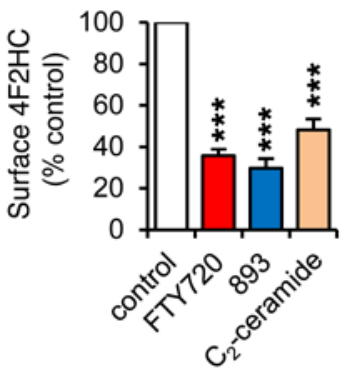

D
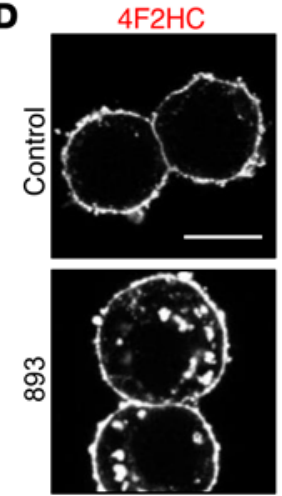

ASCT2
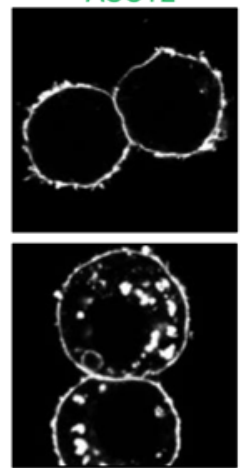

Merged
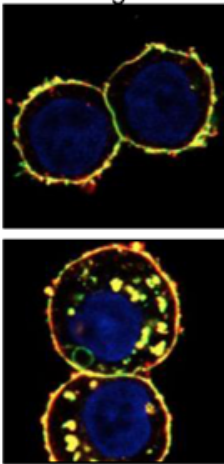
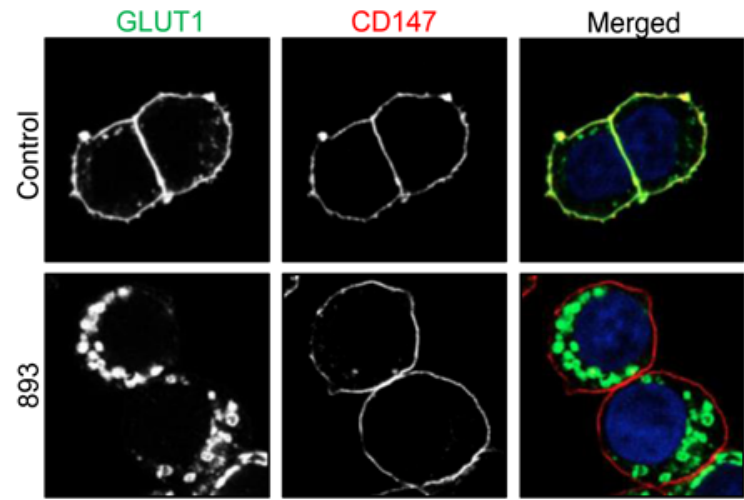

E

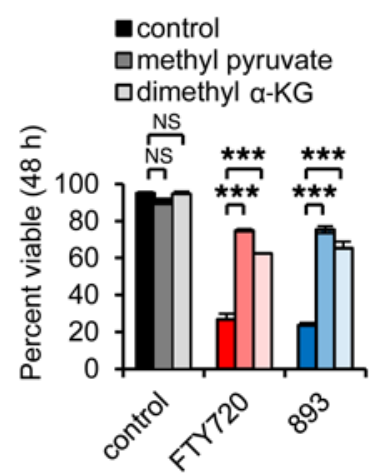

$\mathbf{F}$

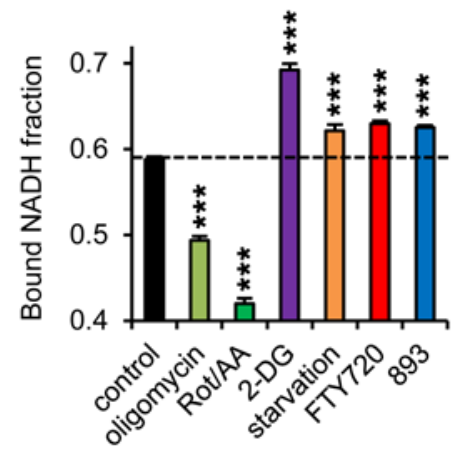

G

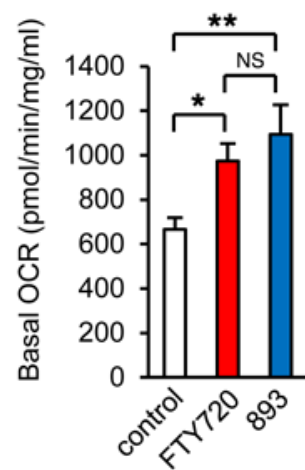

Figure 1. SH-BC-893 triggers nutrient transporter internalization mimicking starvation. (A) Structures of FTY720 and SH-BC-893. (B) S1P, receptor-driven GFP expression measured by flow cytometry in reporter MEFs after a 24-hour incubation with the indicated compounds at 2.5 $\mu \mathrm{M}$. 893, SH-BC-893; 893-P, (SH-BC-893-phosphate); FTY720-P, (FTY720-phosphate). (C) Surface 4F2HC measured by flow cytometry in FL5.12 cells treated with $5 \mu$ M FTY720 or SH-BC-893 or $10 \mu \mathrm{MC}_{2}$-ceramide. (D) SH-BC-893-treated SW620 cells stained as indicated. Scale bar: $10 \mu \mathrm{m}$. (E) Viability of BCLXL-overexpressing FL5.12 cells treated as indicated, with or without $5.5 \mathrm{mM}$ methyl pyruvate or $2 \mathrm{mM}$ dimethyl $\alpha$-ketoglutarate ( $\alpha$-KC). (F) Bound NADH fraction in MEFs treated with mitochondrial inhibitors ( $1 \mu \mathrm{M}$ oligomycin or $1 \mu \mathrm{M}$ rotenone and antimycin A [Rot/AA]), $1 \mathrm{mM} 2-\mathrm{DC}, 5 \mu \mathrm{M}$ FTY720, or $7.5 \mu \mathrm{M} \mathrm{SH-BC-893} \mathrm{for}$ 16 hours. For starvation, growth media were replaced with DMEM lacking glucose and amino acids, supplemented with $10 \%$ dialyzed FCS. Statistical significance was compared with respective controls. (G) Oxygen consumption rate (OCR) in MEFs treated with FTY720 or SH-BC-893 for 16 hours measured by an XF24 Extracellular Flux Analyzer (Seahorse Bioscience). Error bars indicate the mean \pm SEM. ${ }^{*} P<0.05,{ }^{* *} P<0.01$, and ${ }^{* * *} P<0.001$, by unpaired, 2 -tailed Student's $t$ test. $P$ values were determined using Tukey's method when correcting for multiple comparisons. Data are representative of at least 3 independent experiments.

The identification of compounds with good pharmacological properties that restrict access to multiple nutrients presents a significant challenge (17). Sphingolipids offer a promising alternative to competitive inhibitors of individual nutrient uptake pathways. Natural and synthetic sphingolipids limit glucose and amino acid transporter surface expression through evolutionarily conserved effects on membrane trafficking (18-20). Unfortunately, most sphingolipids have significant pharmacological liabilities that prevent their use in patients with cancer, despite their activity in vitro and in animal models $(21,22)$. Even if sphingolipids with acceptable drug properties were developed, lysosomal nutrient generation from macropinosome and/or autophagosome degradation could afford resistance, particularly in tumors with activated Ras, in which these pathways are upregulated $(9,23)$. Here, we report that SH-BC-893, a pharmacologically viable synthetic sphingolipid, is an apical inhibitor of cancer metabolism that blocks nutrient access through multiple, parallel pathways by altering membrane trafficking. 
A
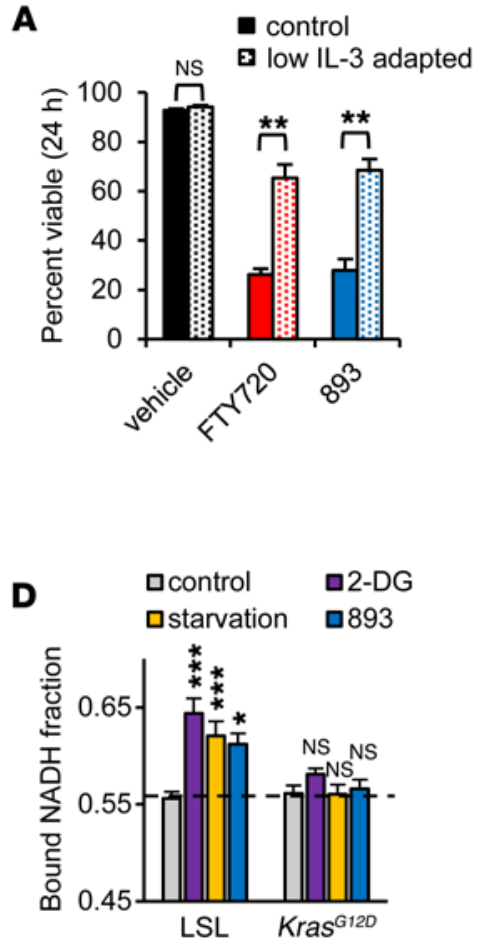

G

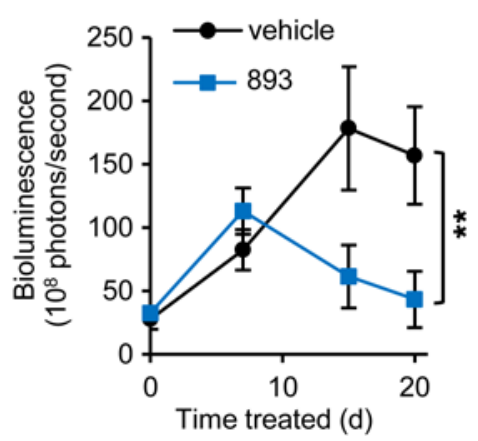

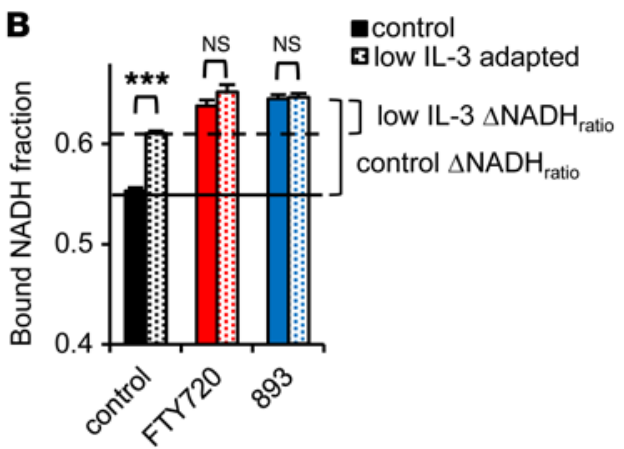
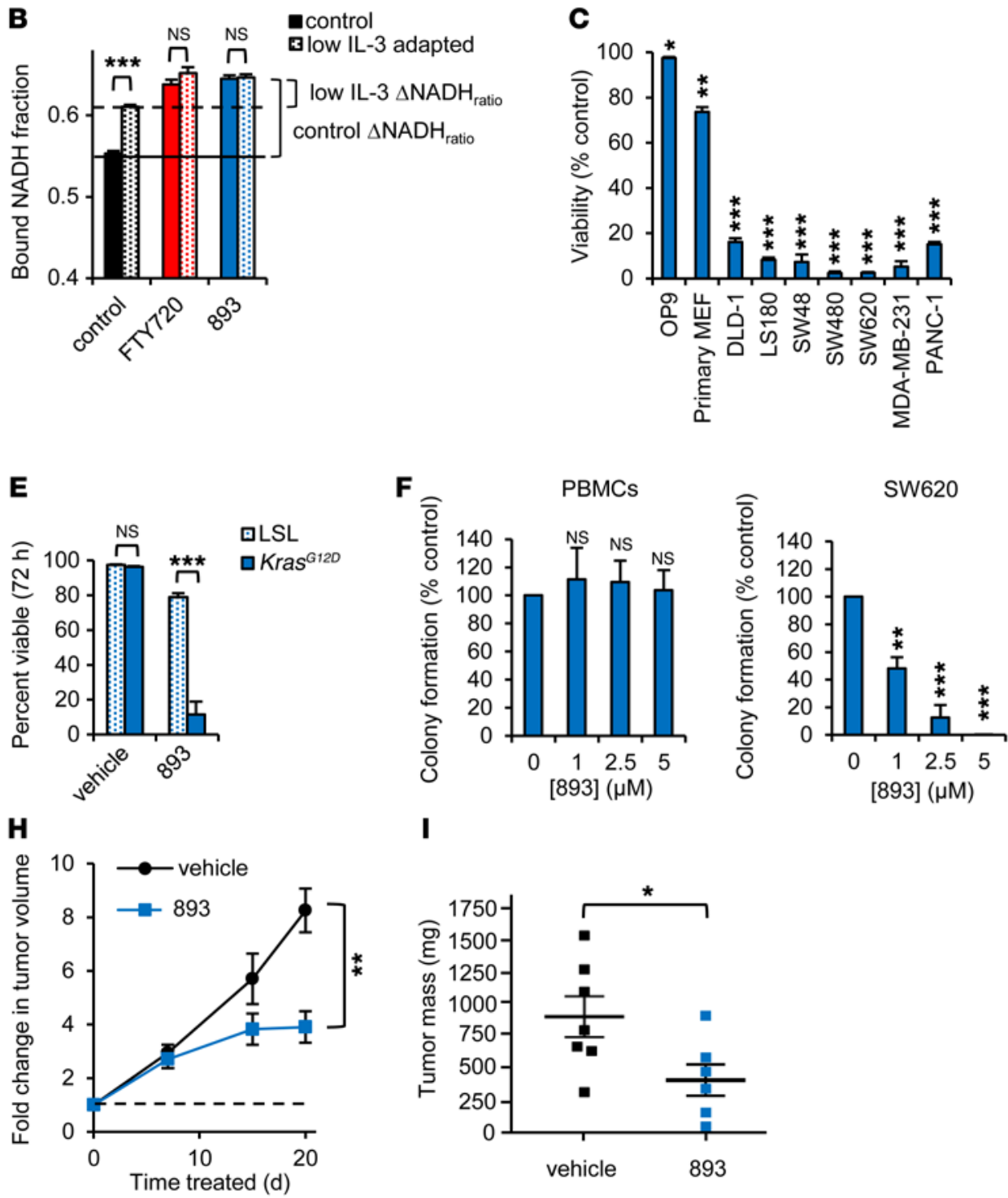

I

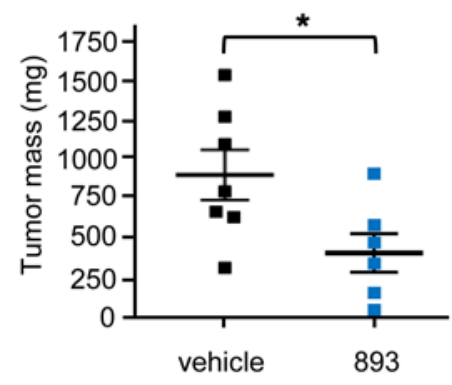

Figure 2. SH-BC-893 selectively kills cancer cells. (A) Viability of FL5.12 cells cultured in control medium ( $500 \mathrm{pg} / \mathrm{ml}$ IL-3) or adapted to low IL-3 (25 pg/ml) treated with $5 \mu \mathrm{M}$ FTY720 or SH-BC-893. (B) Bound NADH fraction in FL5.12 cells cultured as in A and treated with FTY720 or SH-BC-893. (C) Viability of the indicated cells treated with $5 \mu \mathrm{M} \mathrm{SH}-\mathrm{BC}-893$ for 72 hours. Statistical significance was compared with vehicle. OPg (murine bone marrow stromal cells), and primary MEF were nontransformed cells. DLD-1, LS180, SW480, SW480, and SW620 were colon cancer cell lines. MDA-MB-231 and PANC-1 were breast cancer and pancreatic cancer cell lines, respectively. (D) Bound NADH fraction in control or Kras ${ }^{\mathrm{G} 120}$-expressing MEFs treated with $1 \mathrm{mM} 2$-DC, starvation media (glucose and amino acids supplemented with $10 \%$ dialyzed F(S), or $7.5 \mu \mathrm{M} \mathrm{SH}-\mathrm{BC}-893$ for 16 hours. Statistical significance was compared with respective controls. (E) Viability of $p 53^{-1-}$ MEFs, with or without $K$ ras $^{C 12 D}$ expression, that were treated with $6 \mu \mathrm{M} \mathrm{SH}-\mathrm{BC}-893$. (F) Colony formation by normal human PBMCs or SW620 CRC cells, with or without SH-BC-893 at the indicated doses. Error bars indicate the mean \pm SEM. (G-I) Subcutaneous SW620 tumor growth as measured by bioluminescence imaging (BLI) (G), calipers $(\mathbf{H})$, or tumor weight at sacrifice (I). Because FTY720 and SH-BC-893 were similarly potent in vitro (Figure $1, \mathrm{C}$ and E) (26), SH-BC-893 was given i.p. at a dose of $10 \mathrm{mg} / \mathrm{kg}$ for 7 days. On the basis of BLI (C), the dose was increased to $20 \mathrm{mg} / \mathrm{kg}$ on day 8 . Error bars indicate the mean $\pm \mathrm{SD} . n=7$ mice per group. ${ }^{*} P<0.05$, ${ }^{* *} P<0.01$, and ${ }^{* *} P<0.001$, by unpaired, 2 -tailed Student's $t$ test. $P$ values were determined using Tukey's method when correcting for multiple comparisons. In vitro data are representative of at least 3 independent experiments.

\section{Results}

SH-BC-893 starves cancer cells to death. At higher doses than required for immunosuppression, the US Food and Drug Administrationapproved (FDA-approved) multiple sclerosis therapy FTY720 selectively kills cancer cells in vitro and in vivo in part by triggering the internalization of glucose and amino acid transporters (20, 24-26). Unfortunately, FTY720 cannot be repurposed for use in patients with cancer, because it dramatically slows the heart rate at the antineoplastic dose by activating sphingosine-1-phosphate ( $\left.\mathrm{S}_{1} \mathrm{P}_{1}\right)$ receptor 1 in the heart $(22,27,28)$. The conformationally constrained FTY720 analog SH-BC-893, in contrast, could be used therapeutically, as it does not activate $\mathrm{S1P}_{1}$ in reporter cells (Figure 1, A and B). Moreover, neither SH-BC-893 nor its phosphate triggers lymphocyte sequestration, an S1P-dependent effect (27). Importantly, SH-BC-893 still triggers the selective internalization of amino acid $(4 \mathrm{~F} 2 \mathrm{HC}$, also known as SLC3A2, or ASCT2, also known as SLC1A5) and glucose (GLUT1, also known as SLC2A1) transporters (17, 27) (Figure 1, C and D). Other surface proteins, such as CD147, a chaperone protein with 

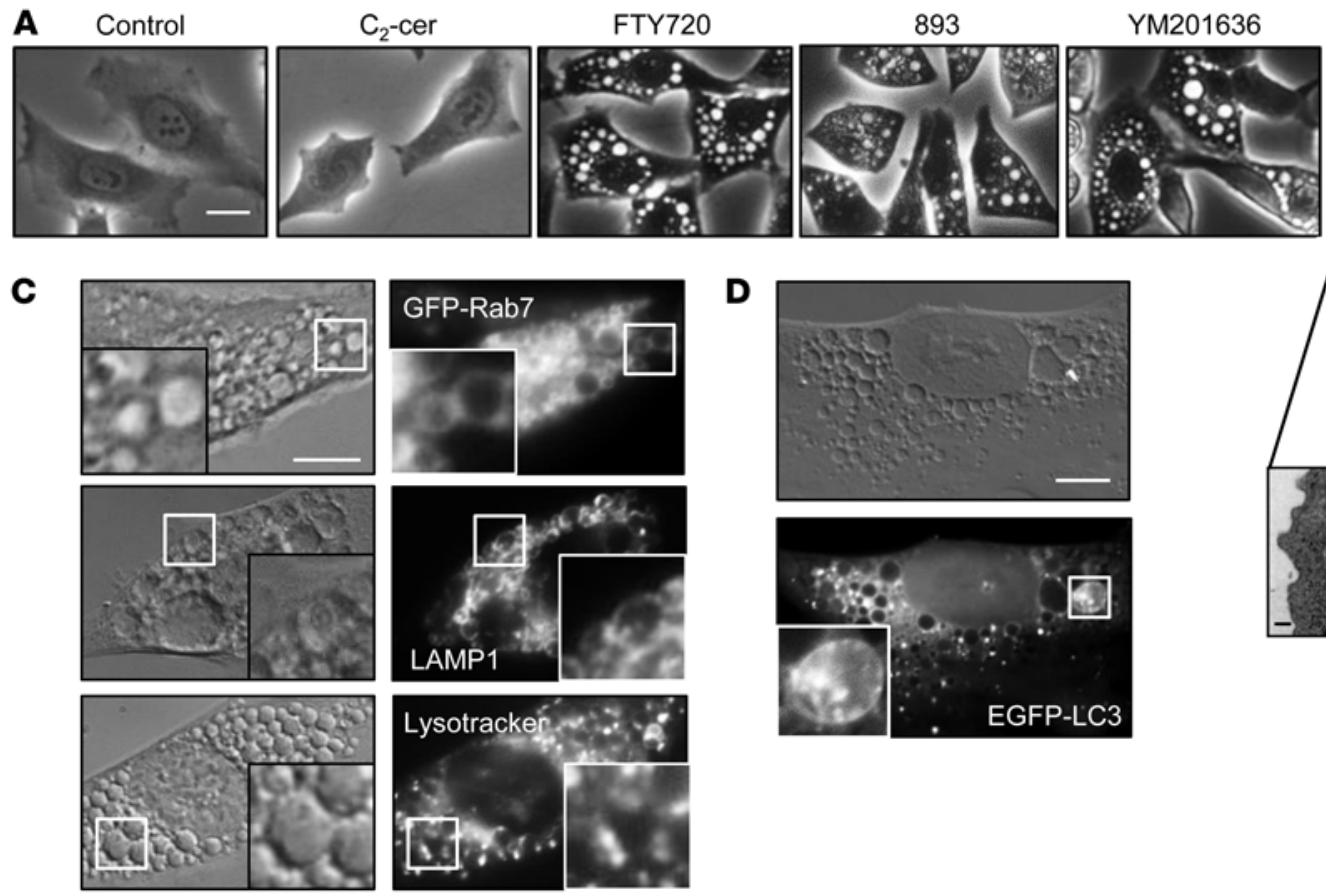

D
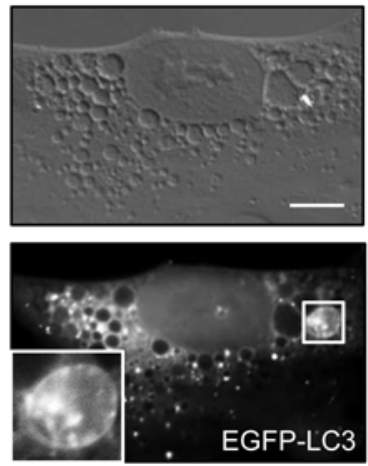

B

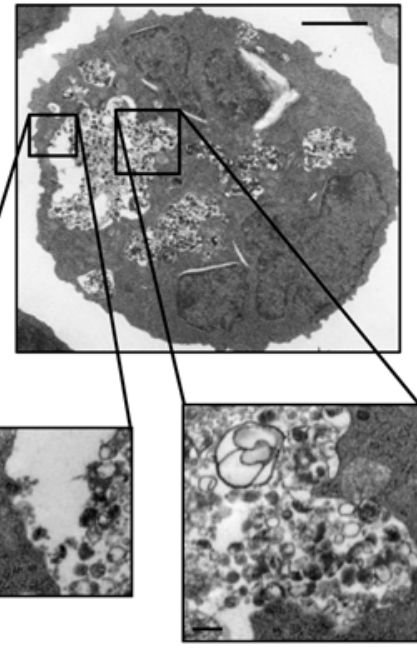

E
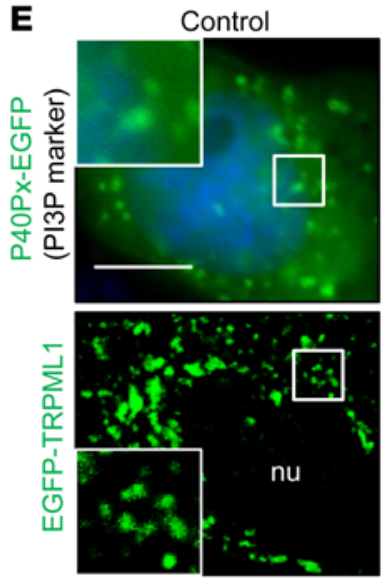
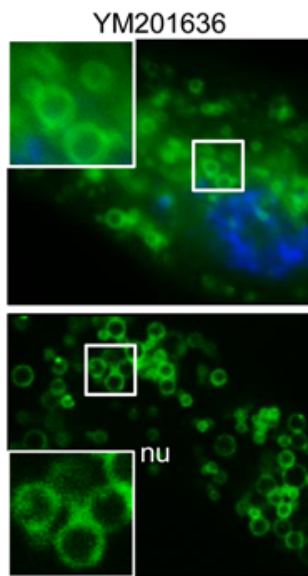

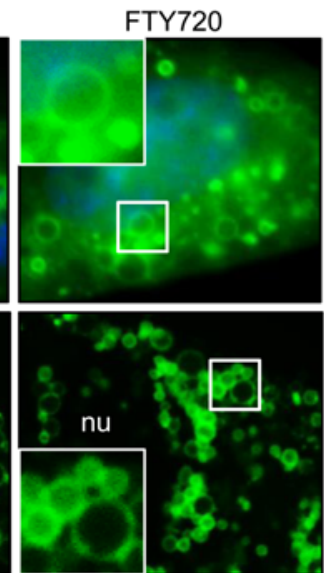

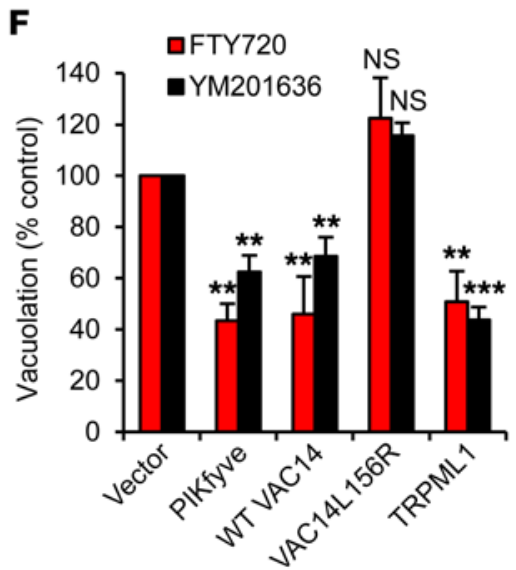

Figure 3. Sphingolipid-induced vacuolation resembles PIKfyve inhibition. (A) HeLa cells treated as indicated with $25 \mu \mathrm{M} \mathrm{C}_{2}$-ceramide, $5 \mu \mathrm{M}$ FTY720 or SH-BC-893, or $800 \mathrm{nM}$ YM201636 for 6 hours. (B) Electron micrograph of an FL5.12 cell treated with FTY720 for 24 hours. Scale bars: $2 \mu \mathrm{m}, 0.2 \mu \mathrm{m}$ in zoom (insets). (C) GFP-RAB7-expressing HeLa cells (top) or MEFs treated with FTY720 and stained as indicated. Images of 3 separate cells are shown. Scale bar: $10 \mu \mathrm{m} ; \times 2.5$ magnification. (D) EGFP-LC3-expressing MEFs treated with FTY720. Scale bar: $10 \mu \mathrm{m} ; \times 2.75$ magnification. (E) P4OPx-EGFP- or EGFP-TRPML1expressing HeLa cells treated with YM201636 or FTY720 for 6 hours. Scale bar: $10 \mu \mathrm{m} ; \times 2.5$ magnification. nu, nucleus. (F) HeLa cells overexpressing PIKfyve, WT VAC14, mutant (L156R) VAC14, or TRPML1 treated with YM201636 or FTY720. Error bars indicate the mean \pm SEM. $n \geq 3$ independent experiments; $\geq 30$ cells per condition were analyzed per experiment. Statistical significance was compared with respective controls. ${ }^{* *} P<0.01$ and ${ }^{* * *} P<0.001$, by unpaired, 2 -tailed Student's $t$ test. $P$ values were determined using Tukey's method to correct for multiple comparisons.

functions similar to those of $4 \mathrm{~F} 2 \mathrm{HC}$, were not affected. As expected, if cells were nutrient limited, blocking apoptosis via BCLXL overexpression did not prevent SH-BC-893-induced cell death (Figure 1E and Supplemental Figure 1, A and B; supplemental material available online with this article; doi:10.1172/JCI87148DS1). In contrast, the transporter-independent, membrane-permeant nutrients methyl pyruvate and dimethyl- $\alpha$-ketoglutarate rescued SH-BC-893-treated cells (Figure 1E). These results confirm that, like FTY720 (20), SH-BC-893 kills cells by limiting nutrient access.

Cells adapt to nutrient limitation by increasing oxidative phosphorylation (29). The relative rate of glycolysis and oxidative phos- phorylation can be monitored by measuring the fluorescence lifetime of NADH (30). A higher ratio of protein-bound to free NADH (increased lifetime) correlates with increased oxidative phosphorylation in multiple cell types, both in vitro and in vivo (31-36). As expected, cells treated with oligomycin or rotenone/antimycin A compensated for the loss of oxidative phosphorylation by increasing glycolysis, thereby reducing the bound NADH fraction (Figure $1 F)$. Conversely, the glycolysis inhibitor 2-deoxy-glucose (2-DG) or glucose and amino acid deprivation increased oxidative phosphorylation and the bound-to-free NADH ratio. As predicted, SH-BC-893 mimicked the effect of amino acid and glucose star- 
A

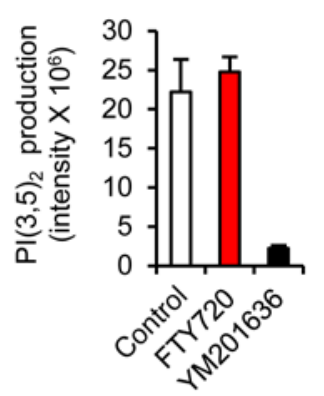

C
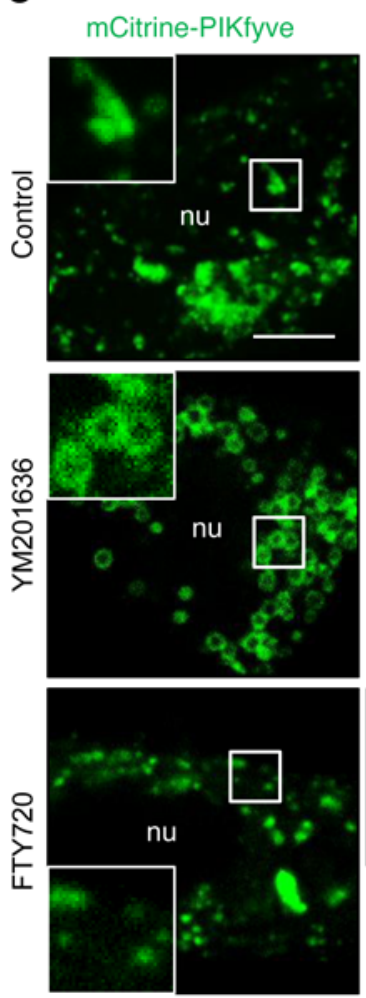

B

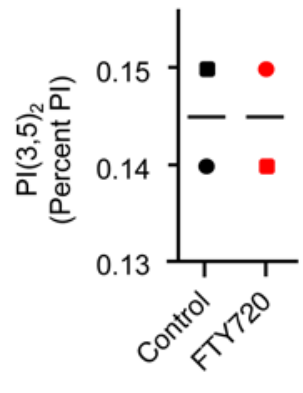

Endogenous PIKfyve/VAC14
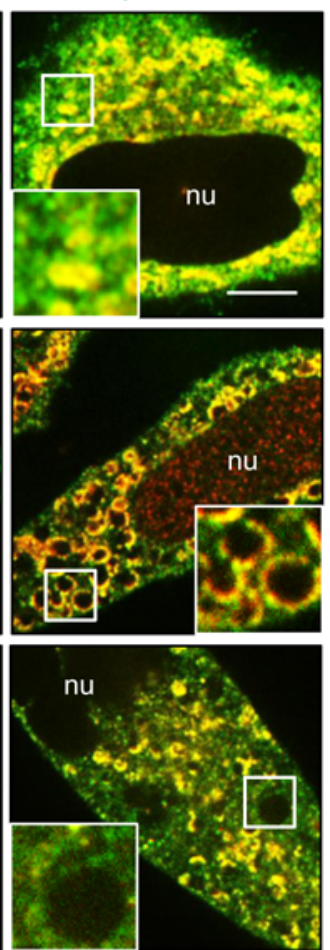

D

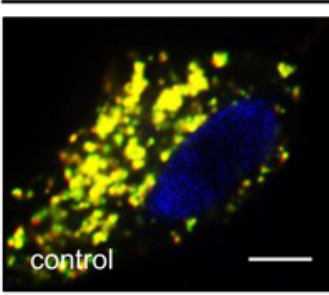

mCherry-TRPML1 and mCitrine-PIKfyve
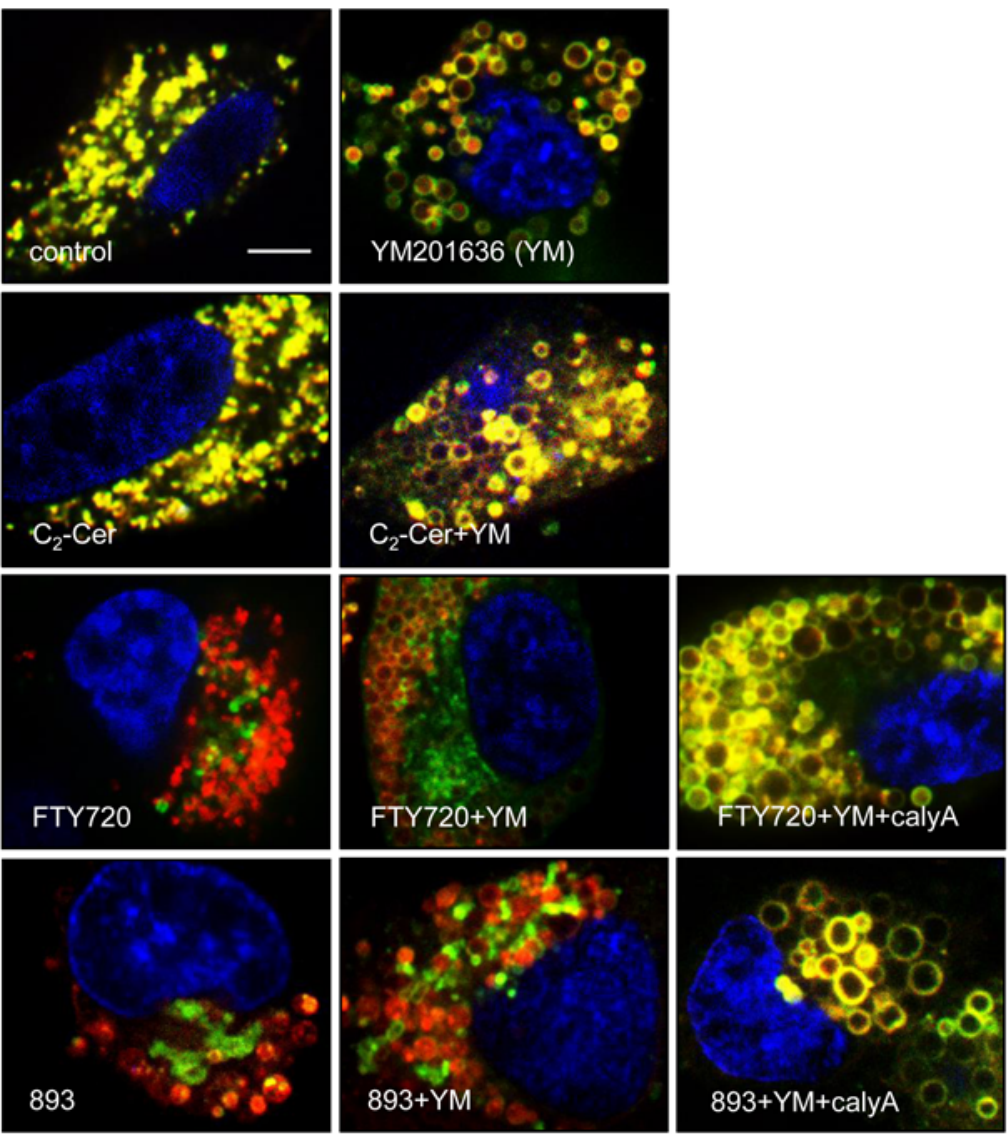

Figure 4. SH-BC-893 and FTY720 disrupt PIKfyve localization but not its activity. (A) In vitro kinase assay with purified FLAG-PIKfyve, with or without $5 \mu$ M FTY720 or 800 nM YM201636. Error bars indicate the mean \pm SD from 3 technical replicates. (B) $\mathrm{PI}(3,5) \mathrm{P}_{2}$ levels in HeLa cells treated with FTY720 expressed as a percentage of total phosphatidylinositol (PI). Bars indicate the average from 2 independent experiments. (C) mCitrine-PIKfyve or endogenous PIKfyve/VAC14 in HeLa cells treated with 800 nM YM201636 or $5 \mu$ M FTY720 for 6 hours. Scale bars: $15 \mu$ M; $\times 2.5$ magnification (C and D). (D) HeLa cells expressing low levels of mCitrine-PIKfyve and mCherry-TRPML1 treated as indicated: $800 \mathrm{nM}$ YM201636, $5 \mu$ M FTY720 or SH-BC-893, $25 \mu \mathrm{M} \mathrm{C} \mathrm{C}_{2}$-ceramide $\left(C_{2}\right.$-Cer), or $5 \mathrm{nM}$ calyculin $\mathrm{A}$ (calyA). Scale bar: $10 \mu \mathrm{m}$.

vation, increasing the bound NADH fraction and cellular oxygen consumption (Figure 1, F and G). Cells responded similarly to the parent compound FTY720. Thus, the metabolic changes triggered by $\mathrm{SH}-\mathrm{BC}-893$ parallel those seen in cells with restricted access to key metabolic substrates.

If SH-BC-893 kills cells by limiting nutrient access, cells with a higher anabolic rate should be more sensitive. The anabolic rate of murine FL5.12 cells can be titrated by modulating the levels of their required growth factor IL-3 (37); comparing FL5.12 cells grown in high and low IL-3 concentrations allows the impact of elevated growth factor signaling and anabolism to be evaluated in a constant genetic background. High concentrations of IL-3 drive aerobic glycolysis and a rapid doubling time $(12 \mathrm{~h})$. Reducing IL-3 levels slows proliferation and increases oxidative phosphorylation without compromising cell viability (Figure 2, A and B) (37). As predicted, maintenance in low IL-3 medium reduced the need for metabolic adaptation (Figure 2B) and protected cells from SH-BC-893-induced death (Figure 2A), suggesting that elevated growth factor signaling and anabolism in transformed cells will confer hypersensitivity to SH-BC-893. Indeed, nontransformed murine OP9 bone marrow stromal cells and primary murine embryonic fibroblasts (MEFs) were less sensitive to SH-BC-893 than were human cancer cell lines (Figure 2C). Many of these cancer cell lines carry activating mutations in Ras. In fact, KRAS activation following Cre expression in Lox-STOP-Lox-Kras ${ }^{G 12 D}$ (LSL$\mathrm{Kras}^{\mathrm{G} 12 D}$ ) MEFs (38) was sufficient to limit metabolic flexibility and 
A
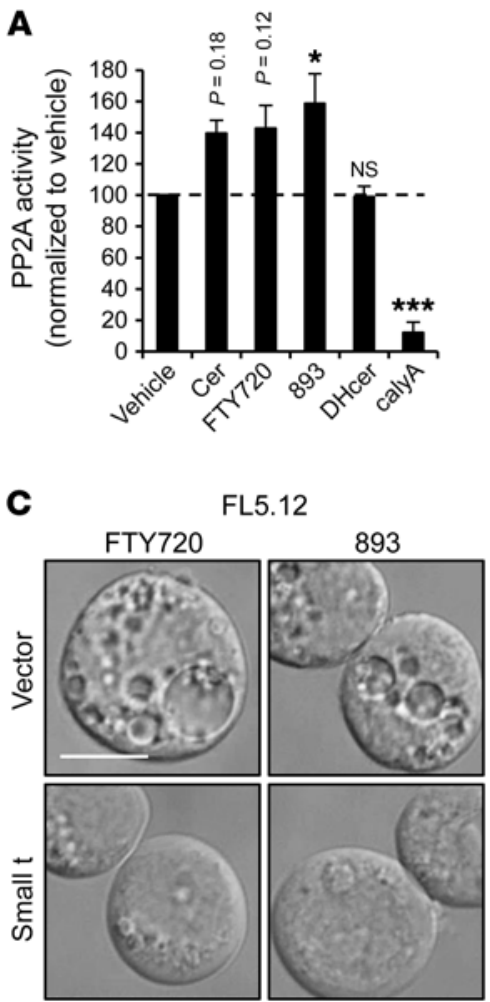

B

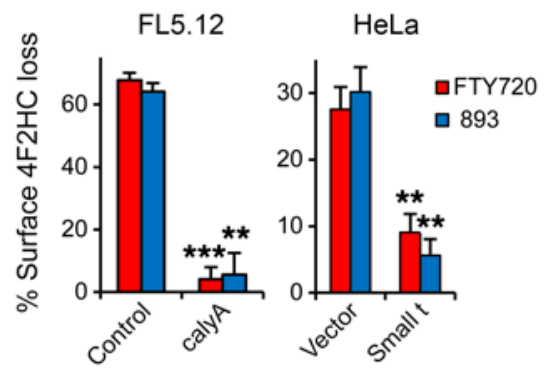

HeLa

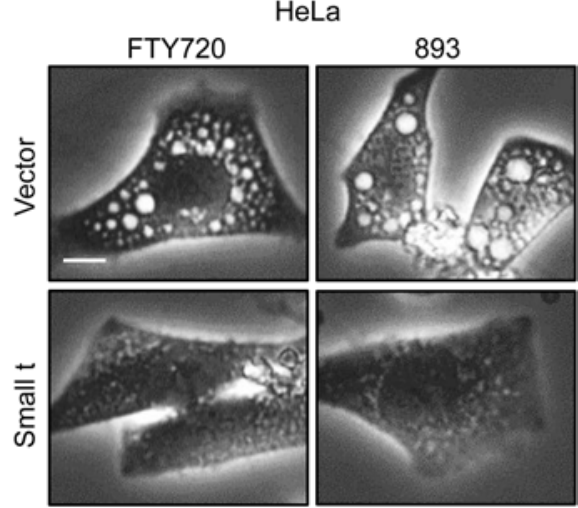

Figure 5. SH-BC-893 and FTY720 activate PP2A to induce vacuolation. (A) PP2A phosphatase activity was measured in FL5.12 cell lysates in the presence or absence of $50 \mu \mathrm{M} \mathrm{C} C_{2}$-ceramide (cer) or dihydro- $C_{2}$ ceramide (DHcer), $5 \mu \mathrm{M}$ FTY720 or SH-BC-893 (893), or $5 \mathrm{nM}$ calyculin A. (B and C) Surface $4 \mathrm{~F} 2 \mathrm{HC}$ (B) or vacuolation (C) in FL5.12 cells pretreated with vehicle or $5 \mathrm{nM}$ calyculin A or HeLa cells expressing vector or SV40 small t antigen treated as indicated. Error bars indicate the mean \pm SEM. ${ }^{*} P<0.05$, ${ }^{* *} P<0.01$, and ${ }^{* * *} P<0.001$, by unpaired, 2-tailed Student's $t$ test. $P$ values were determined using Tukey's method when correcting for multiple comparisons. Data are representative of at least 3 independent experiments. Scale bars: $10 \mu \mathrm{m}$. sensitize cells to SH-BC-893 (Figure 2, D and E). Loss of the tumor suppressor PTEN produced similar effects (Supplemental Figure 2, A and B). Importantly, oncogenic mutations did not affect surface nutrient transporter downregulation in $\mathrm{Kras}^{G 12 D}$-expressing or PTEN-deficient MEFs (Supplemental Figure 2C). These results indicate that both normal and transformed cells express the SH-BC-893 target, and differential sensitivity to the compound stems from the lack of metabolic adaptation in cancer cells. Consistent with these results in MEFs, normal human peripheral blood mononuclear cells (PBMCs) were also resistant to SH-BC-893 relative to SW620 colon cancer cells (Figure 2F) in colony formation assays. Taken together, these data suggest that constitutive anabolism sensitizes cancer cells to SH-BC-893 and could generate an acceptable therapeutic index.

To determine whether SH-BC-893 inhibited tumor growth in vivo, luciferase-expressing SW620 xenografts were generated. Bioluminescence, caliper measurements, and tumor mass at sacrifice were reduced to a similar degree by SH-BC-893 and FTY720 (Figure 2, G-I and Supplemental Figure 2D). Consistent with these effects, SH-BC-893 was present at low micromolar concentrations in both tumors and plasma at sacrifice (Supplemental Figure 2E). Mild weight loss occurred in treated mice, as expected, given that nutrient access would be restricted in both normal and transformed cells (Supplemental Figure 2F). These results suggest that $\mathrm{SH}-\mathrm{BC}-893$ could provide a safe and effective means to target Ras-driven cancer anabolism in vivo.

SH-BC-893 interferes with late endocytic trafficking. SH-BC-893 was surprisingly effective, given that Ras activation increases macropinocytosis and autophagy $(9,23)$, processes that should provide resistance to surface nutrient transporter loss. We therefore hypothesized that SH-BC-893 might affect additional traf- ficking pathways. Interestingly, SH-BC-893 induced equally striking cytosolic vacuolation in both nontransformed and cancer cells (Figure 3A and Supplemental Figure 3, A-C). These vacuoles contained intraluminal vesicles (ILVs) as well as amorphous, partially degraded material, suggesting that they originate from multivesicular bodies (MVBs) or another late endocytic compartment (Figure 3B). Vacuoles were positive for the late endosomal markers LAMP1 and RAB7 (Figure 3C) and negative for the early endosomal markers EEA1 and RAB5 and the lipid stain Nile red (Supplemental Figure 3D). Acidified puncta, probably lysosomes, were observed within or proximal to vacuoles along with material marked as autophagosomes by GFP-LC3 (Figure 3, C and D, and Supplemental Figure 3E). Taken together, these results suggest that SH-BC-893 enlarges MVBs.

$\mathrm{PI}(3,5) \mathrm{P}_{2}$, the product of the PI3P 5-kinase PIKfyve, regulates membrane fusion and ILV formation in MVBs (39). Reducing PIKfyve activity with the inhibitor YM201636 produced PI3P-positive, $\mathrm{PI}(3,5) \mathrm{P}_{2}$-negative vacuoles that were phenotypically similar to those generated by SH-BC-893 and FTY720 (Figure 3, A and E, and Supplemental Figure 3, A and F) (40-42). The $\mathrm{Ca}^{2+}$ channel TRPML1 (transient receptor potential cation channel, mucolipin subfamily, member 1) is found in MVB membranes, where it is activated by $\mathrm{PI}(3,5) \mathrm{P}_{2}$, generated by PIKfyve (43). TRPML1 accumulated in vacuolar membranes in FTY720- and YM201636-treated cells (Figure 3E). Overexpression of PIKfyve, its scaffolding protein VAC14, or its effector protein TRPML1 rescued either FTY720- or YM201636-induced vacuolation, while a VAC14 mutant that does not associate with PIKfyve was ineffective at preventing vacuolation (Figure $3 \mathrm{~F}$ ). Together, these data suggest that SH-BC-893 and FTY720 enlarge MVBs by reducing PIKfyve activity to an extent similar to that of YM201636. 
A
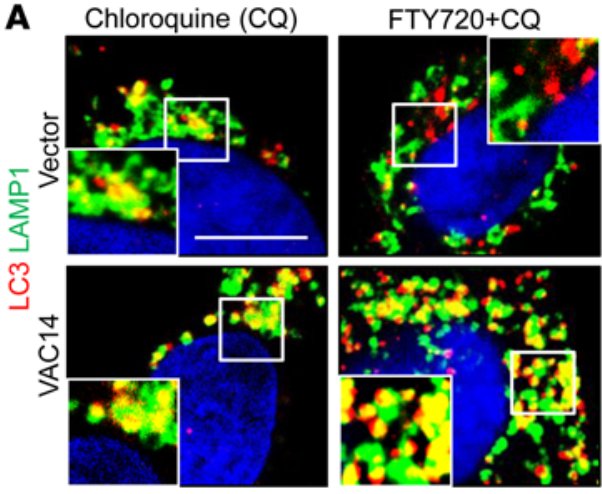

D
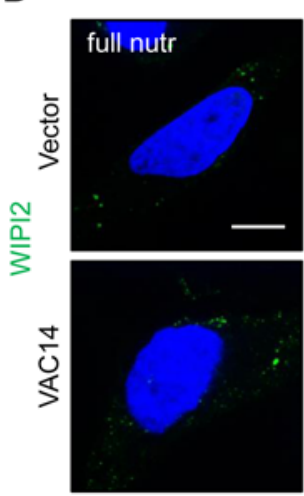

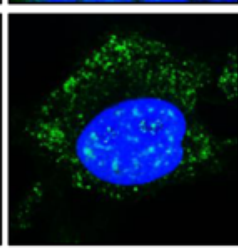

Low nutrients

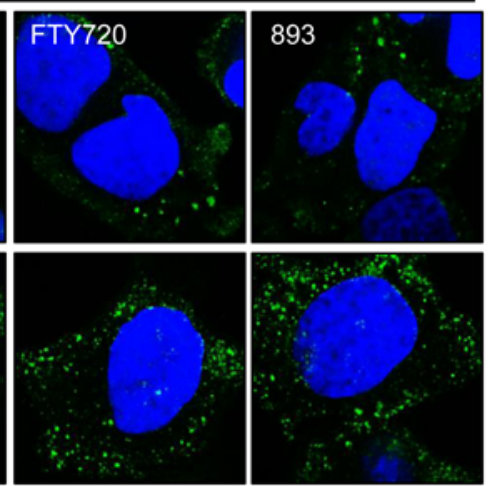

$893+C Q$
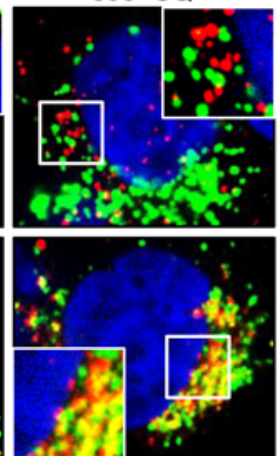

$\mathrm{YM}+\mathrm{CQ}$

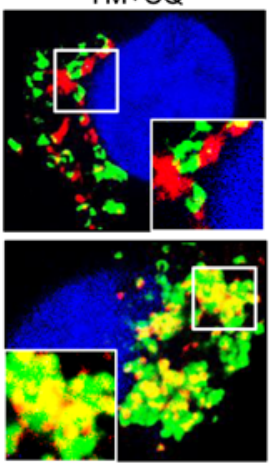

B

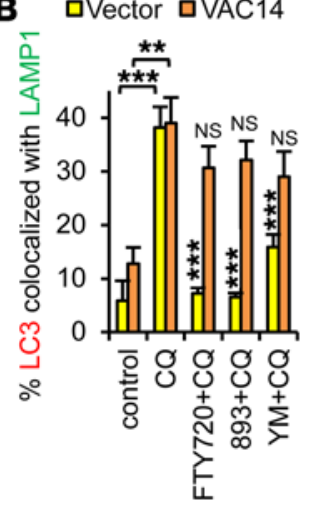

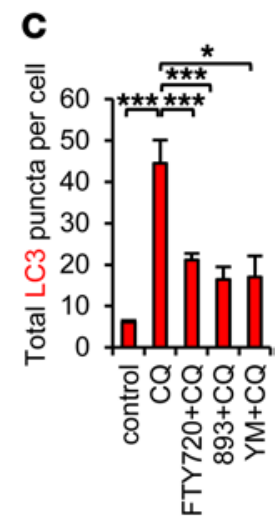

E

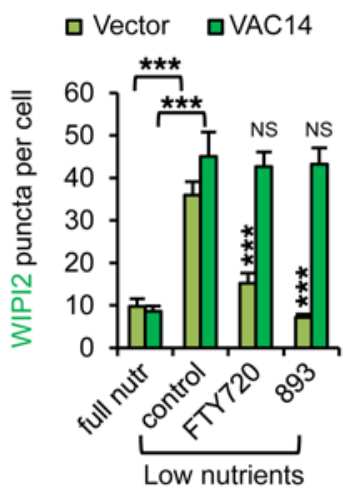

F

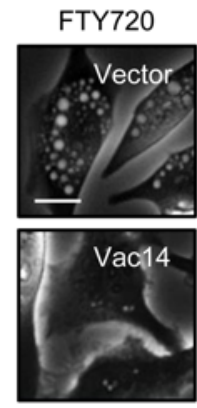

G

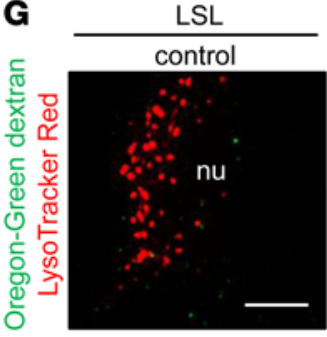

Kras $^{G 12 D}$

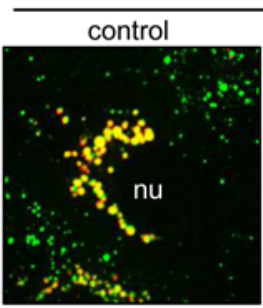

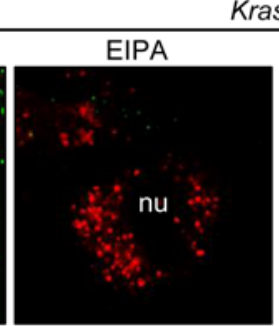

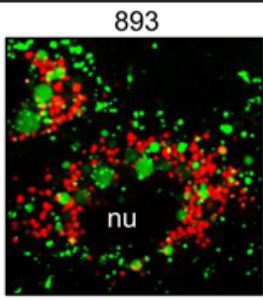

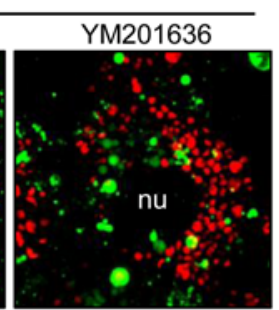

H

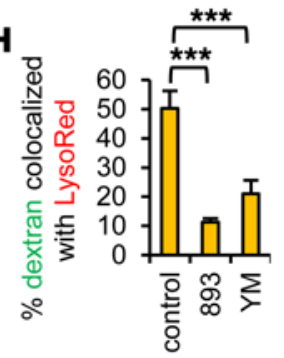

Figure 6. SH-BC-893 reduces autophagic flux and macropinosome degradation. (A) Control or VAC14-overexpressing HeLa cells treated with $25 \mu \mathrm{M}$ chloroquine (CQ), with or without $5 \mu \mathrm{M} \mathrm{FTY720,} \mathrm{were} \mathrm{stained} \mathrm{for} \mathrm{LC3} \mathrm{and} \mathrm{LAMP1} \mathrm{and} \mathrm{evaluated} \mathrm{by} \mathrm{confocal} \mathrm{microscopy.} \mathrm{(B} \mathrm{and} \mathrm{C)} \mathrm{Quantification} \mathrm{of} \mathrm{LC3-LAMP1}$

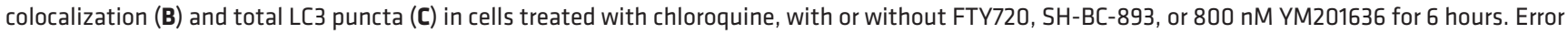
bars indicate the mean \pm SEM. $n \geq 35$ cells evaluated per condition. (D) Vector or VAC14-overexpressing HeLa cells were nutrient stressed in DMEM lacking amino acids and glucose in the presence or absence of $5 \mu \mathrm{M}$ FTY720 or SH-BC-893 and stained for WIPI2. (E) Quantification of WIPI2 puncta. Error bars indicate the mean \pm SEM. $n \geq 50$ cells evaluated per condition. Statistical significance was compared with the respective low-nutrient control unless otherwise indicated. (F) HeLa cells overexpressing VAC14 were treated with $5 \mu$ M FTY720 for 6 hours. (G and H) Dextran uptake in p53 ${ }^{-1-}$ LSL-Kras ${ }^{6120}$ MEFs before (LSL) or after (Kras $\left.{ }^{612 D}\right)$ introduction of Cre, with or without the macropinocytosis inhibitor EIPA, SH-BC-893, or YM201636 (C). Colocalization of dextran and LysoTracker Red $(\mathbf{H})$ was determined using Imagej. Error bars indicate the mean \pm SEM shown. $n \geq 15$ cells evaluated per condition. ${ }^{*} P<0.05,{ }^{* *} P<0.01$, and ${ }^{* *} P<0.001$, by unpaired, 2 -tailed Student's $t$ test. $P$ values were determined using Tukey's method when correcting for multiple comparisons. Scale bars: $20 \mu \mathrm{m}$; magnification in (A) $\times 1.8$. nutr, nutrient; YM, YM201636.

Unexpectedly, FTY720 did not inhibit PIKfyve kinase activity or reduce $\mathrm{PI}(3,5) \mathrm{P}_{2}$ levels at concentrations that profoundly vacuolate cells (Figure 4, A and B). Rather, FTY720 disrupted PIKfyve localization. While mCitrine-tagged PIKfyve localized to the limiting membrane of YM201636-induced vacuoles as expected, PIKfyve was present in clumps between vacuoles in FTY720-treated cells (Figure 4C and Supplemental Figure 3F). Validated antibodies recognizing endogenous PIKfyve and VAC14 confirmed this result (Figure 4C and Supplemental Figure 4, A and B). See complete unedited blots in the supplemental material. Consistent with their disparate mechanisms of action, YM201636 abolished membrane association of the $\mathrm{PI}(3,5) \mathrm{P}_{2}$ probe mCherry-ML1N ${ }^{\star} 2(44)$, while in FTY720- or SH-BC-893-treated cells, mCherry-ML1N ${ }^{\star} 2$ colocalized with PIKfyve to puncta between the vacuoles (Supplemental Figure 3F). PIKfyve-Vac14 association was not altered by FTY720 (Supplemental Figure 4C). Moreover, neither PIKfyve (Figure 4D) nor mCherry-ML1N ${ }^{\star} 2$ (Supplemental Figure 4D) colocalized with the $\mathrm{PI}(3,5) \mathrm{P}_{2}$ effector protein TRPML1 on vacuoles in FTY720- 
A

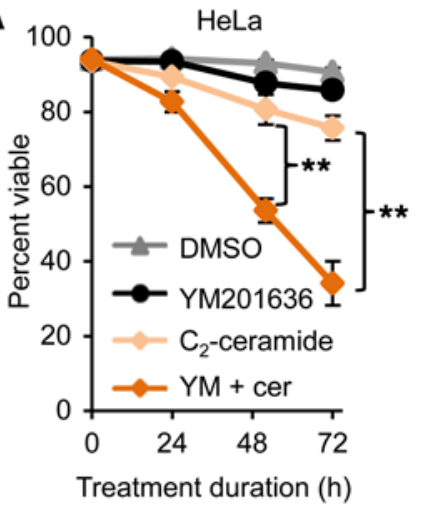

B

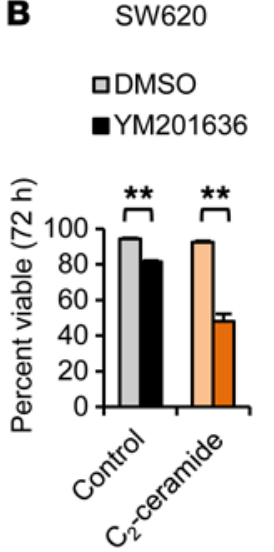

HeLa

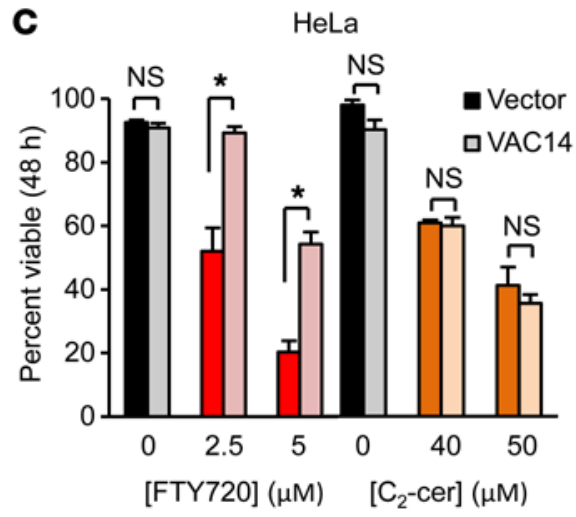

D $\quad$ sw480

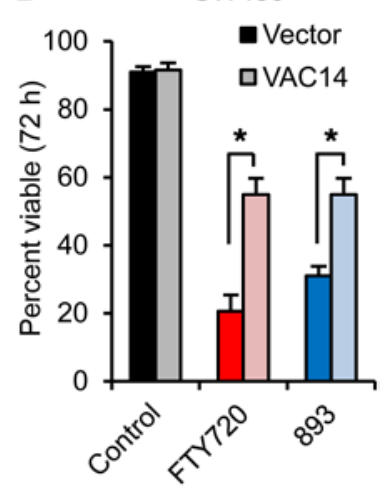

E

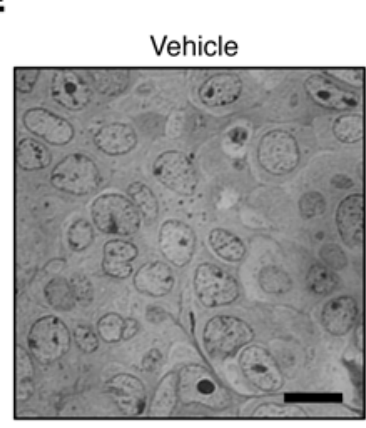

893

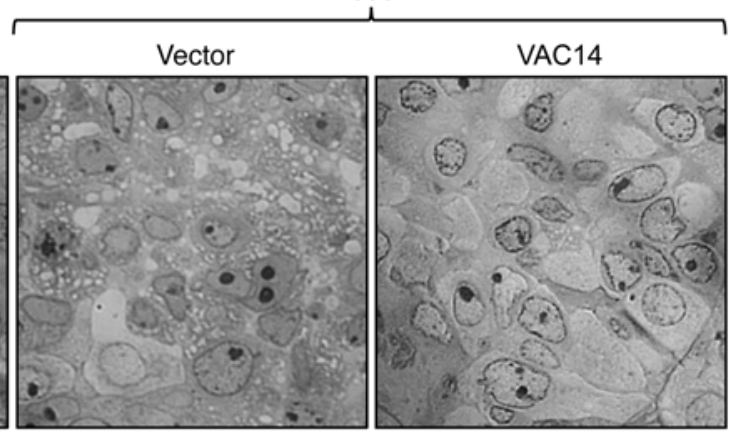

$\mathbf{F}$

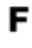

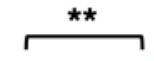

口

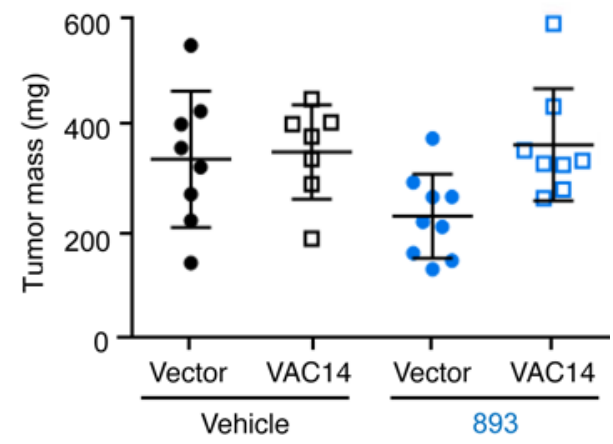

Figure 7. Vacuolation enhances the antineoplastic effects of SH-BC-893 in vitro and in vivo. (A and B) Viability of HeLa (A) or SW620 (B) cells treated with DMSO, 800 nM YM201636, $25 \mu \mathrm{M} \mathrm{C}$-ceramide, or YM201636 plus C -ceramide (YM + cer) for the indicated durations. (C and D) Viability of control or VAC14-overexpressing cells treated with FTY720 or $\mathrm{C}_{2}$-ceramide. Error bars indicate the mean \pm SEM. (E) Vacuolation evaluated by bright-field microscopy in SW480 tumors from mice treated with vehicle (water) or $60 \mathrm{mg} / \mathrm{kg} \mathrm{SH-BC-893} \mathrm{by} \mathrm{gavage} \mathrm{daily} \mathrm{for} 18$ days. Scale bar: $10 \mu \mathrm{m}$. (F) Tumor weight at sacrifice. $P=0.06$ between vehicle and SH-BC-893-treated vector groups (2-tailed Student's $t$ test). Error bars indicate the mean \pm SD. $n \geq 7$ mice per group. ${ }^{*} P<0.05$ and ${ }^{* *} P<0.01$, by unpaired, 2 -tailed Student's $t$ test. In vitro data are representative of at least 3 independent experiments.

or SH-BC-893-treated cells. As TRPML1 was present on both YM201636- and SH-BC-893-induced vacuoles (Figure 3E and Supplemental Figure 4D), PIKfyve, and not TRPML1, was mislocalized by FTY720 and SH-BC-893. FTY720 and SH-BC-893 also eliminated PIKfyve from the TRPML1-positive vacuoles in YM201636-treated cells (Figure 4D). Consistent with its lack of vacuolating activity (Figure 3A), ceramide did not disrupt PIKfyve-TRPML1 colocalization in the presence or absence of YM201636 (Figure 4D). These results indicate that FTY720 and SH-BC-893 induce vacuolation by mislocalizing PIKfyve, leading to generation of the membrane-anchored lipid $\mathrm{PI}(3,5) \mathrm{P}_{2}$ in a compartment separate from its transmembrane effector protein, TRPML1.

Ceramide, FTY720, and SH-BC-893 trigger nutrient transporter loss by activating PP2A (Figure 5, A and B) (18, 20, 27, 45, 46). Activation of PP2A by sphingolipids is specific, as dihydroceramide, which differs from ceramide by a single saturated bond, fails to activate PP2A, does not kill cells, and does not trigger transporter loss or vacuolation (Figure 5A and Supplemental Figure 5A) (46, 47). FTY720 or SH-BC-893 also caused vacuolation by activating PP2A, as the selective PP2A inhibitor calyculin A and the protein inhibitor of PP2A, SV40 small $t$ antigen, both blocked this effect (Figure 5C); YM201636-induced vacuolation was unaffected by PP2A inhibition (Supplemental Figure 5B). PP2A activation trig- gered PIKfyve mislocalization, as inhibiting PP2A restored PIKfyve to YM201636-induced vacuoles in FTY720- and SH-BC-893treated cells (Figure 4D). As ceramide triggers transporter loss by activating PP2A (18) but does not vacuolate cells (Figure 3A) or induce PIKfyve mislocalization (Figure 4D), different PP2A heterotrimers or distinct pools of PP2A promote nutrient transporter downregulation and vacuolation. Consistent with a model in which FTY720 and SH-BC-893 disrupt 2 distinct trafficking pathways downstream of different PP2A complexes, amino acid and glucose transporters internalized by FTY720 and SH-BC-893 did not colocalize with the PIKfyve complex (Supplemental Figure 5C). Moreover, triggering vacuolation with YM201636 did not decrease surface nutrient transporter levels, and preventing vacuolation by overexpressing VAC14 did not interfere with nutrient transporter downregulation by FTY720 (Supplemental Figure 5, D and E). Taken together, these data indicate that FTY720 and SH-BC-893 disrupt PIKfyve localization and nutrient transporter trafficking in both normal and transformed cells through 2 distinct PP2A-dependent mechanisms.

PIKfyve mislocalization blocks lysosomal nutrient production. Cells adapt to nutrient stress by increasing autophagic flux (17). However, autophagosome-lysosome fusion reactions depend upon $\mathrm{Ca}^{2+}$ released through TRPML1 channels that are activated 

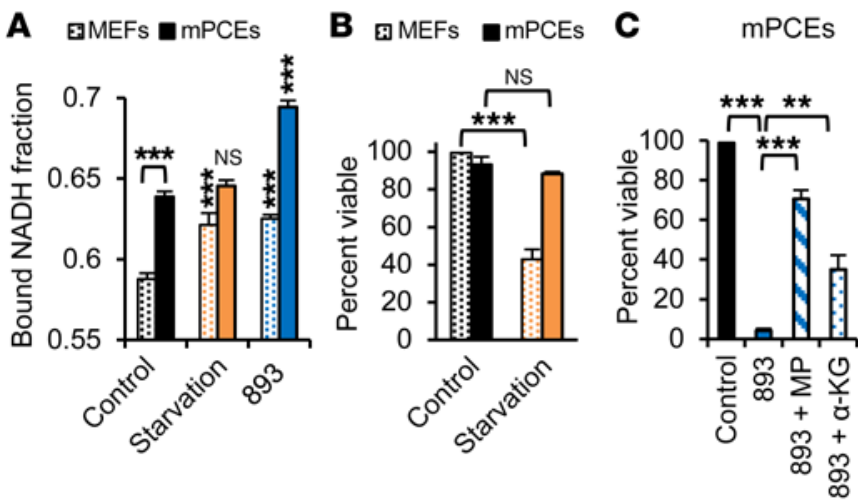
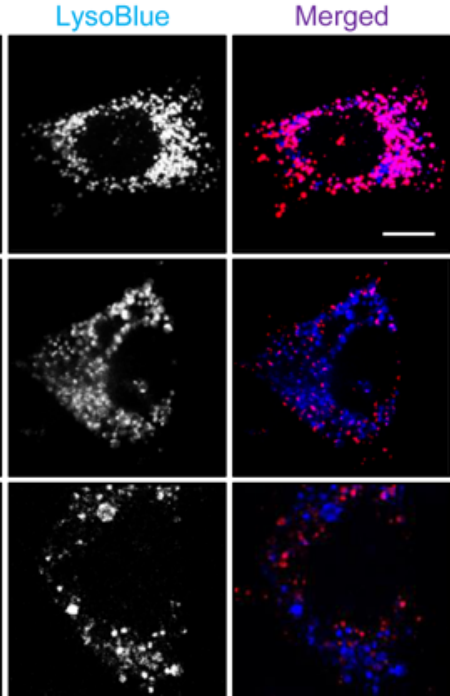

G

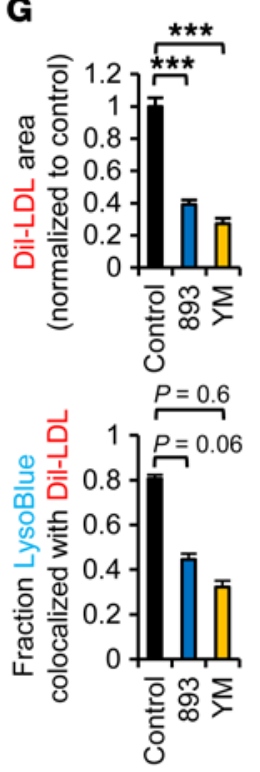

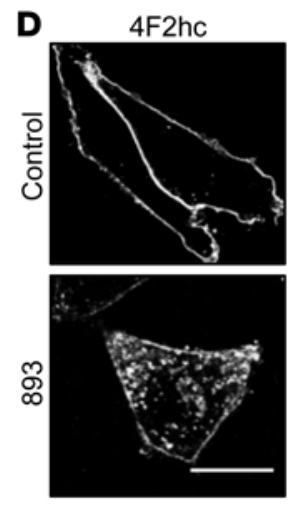

H
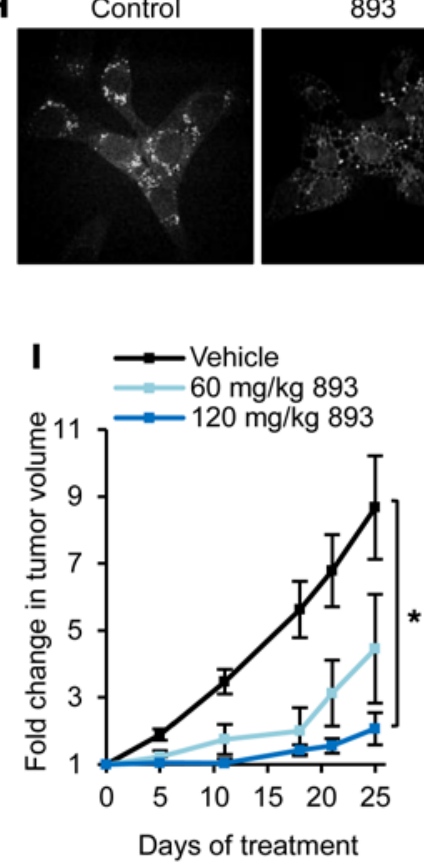

893
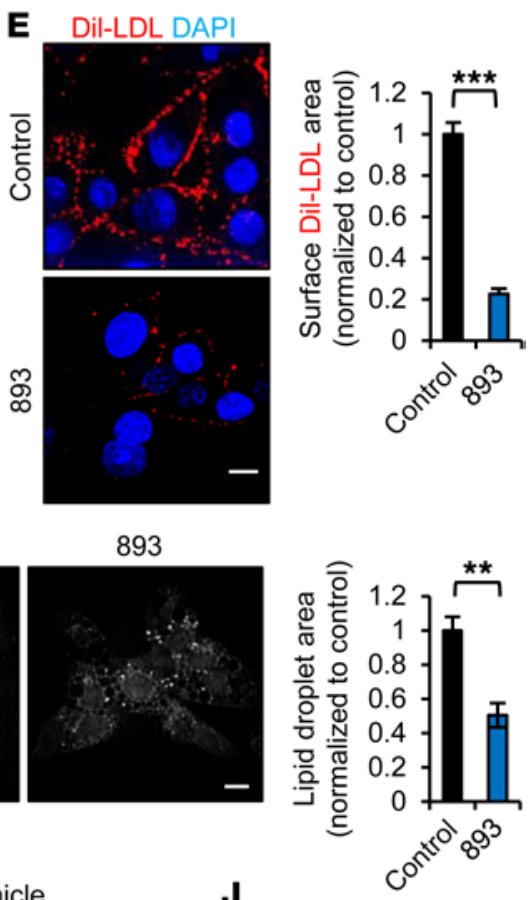

$\mathbf{J}$

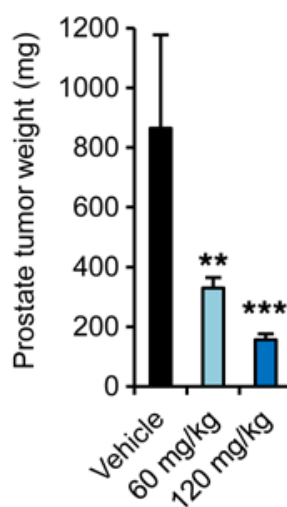

Figure 8. SH-BC-893 starves Pten ${ }^{-/-}$prostate cancer cells. (A) Bound NADH fraction in MEFs or mPCEs. Error bars indicate the mean \pm SEM. $n \geq 50$ cells evaluated per condition. (B) Viability of MEFs or mPCEs in starvation media (DMEM lacking glucose and amino acids supplemented with $10 \%$ FBS). (C) Viability of $\mathrm{mPCEs}$ treated with $5 \mu \mathrm{M} \mathrm{SH}-\mathrm{BC}-893$, with or without $11 \mathrm{mM}$ methyl pyruvate or $7 \mathrm{mM}$ dimethyl $\alpha$-ketoglutarate. Error bars indicate the mean \pm SEM. (D) $\mathrm{mPCEs}$ treated with $5 \mu \mathrm{M}$ SH-BC-893 stained for 4F2HC. (E) Dil-LDL bound to surface LDL receptors of mPCEs maintained at $4^{\circ} \mathrm{C}$. Error bars indicate the mean \pm SEM. $n \geq 80$ cells per condition. (F and $\mathbf{G})$ Dil-LDL uptake and lysosomes in mPCEs treated with SH-BC-893 or YM201636. Quantification of total Dil-LDL area (F) and colocalization of Dil-LDL with LysoTracker Blue (C). Error bars indicate the mean \pm SEM. $n \geq 30$ cells per condition. (H) mPCEs treated with SH-BC-893 for 3 hours were imaged using CARS to detect lipid droplets. Error bars indicate the mean \pm SEM. $n=5$ fields. (I) Growth of mPCE s.c. isografts measured by calipers. $n=7$ per group. (J) Mean weight of autochthonous prostate tumors at sacrifice. $n=9$ for the vehicle-treated and the $60 \mathrm{mg} / \mathrm{kg}$ SH-BC-893 groups; $n=8$ for the 120 $\mathrm{mg} / \mathrm{kg}$ group. Statistical significance was compared with respective controls unless otherwise indicated. ${ }^{*} P<0.05$, ${ }^{* *} P<0.01$, and ${ }^{* * *} P<0.001$, by unpaired, 2-tailed Student's $t$ test (A-C, E, and $\mathbf{G}-\mathbf{I}$ ) and Mann-Whitney $U$ test for comparison of treated mice with controls ( $)$ ). $P$ values were determined using Tukey's method when correcting for multiple comparisons. Scale bars: $20 \mu \mathrm{m}$. LysoBlue, LysoTracker Blue.

by $\mathrm{PI}(3,5) \mathrm{P}_{2}(43,48)$. The loss of PIKfyve and PI $(3,5) \mathrm{P}_{2}$ colocalization with TRPML1 (Figure 4D and Supplemental Figure 4D) suggested that SH-BC-893 might limit autophagic flux. In cells where autophagolysosomes were stabilized by chloroquine, the addition of FTY720, SH-BC-893, or YM201636 reduced the fusion of LC3-positive autophagosomes with LAMP1-positive lysosomes (Figure 6, A and B). Interestingly, vacuolating sphingolipids also decreased the total number of LC3 puncta per cell, suggesting that autophagosome formation was reduced (Figure 6C). PI5P is essential for autophagosome biogenesis upon glucose depletion (49). As PI5P is produced by dephosphorylating $\mathrm{PI}(3,5) \mathrm{P}_{2}(50)$, PI5P might also be mislocalized in SH-BC-893-treated cells, thereby disrupting autophagosome formation. Indeed, FTY720 and SH-BC-893 reduced the number of WIPI2-positive nascent autophagosomes detected in low-nutrient media, without affecting PI5P levels (Figure 6, D and E, and Supplemental Figure 6A). Thus, SH-BC-893 inhibited both autophagosome formation and degradation. VAC14 overexpression limited vacuolation (Figure 3F, Figure 6F, and Supplemental Figure $6 \mathrm{~B}$ ) and should rescue autophagic flux in SH-BC-893-treated cells. VAC14 overexpression restored both autophagosome formation and lysosomal fusion in FTY720- and SH-BC-893-treated cells (Figure 6, A, B, D, and E). Together, these 


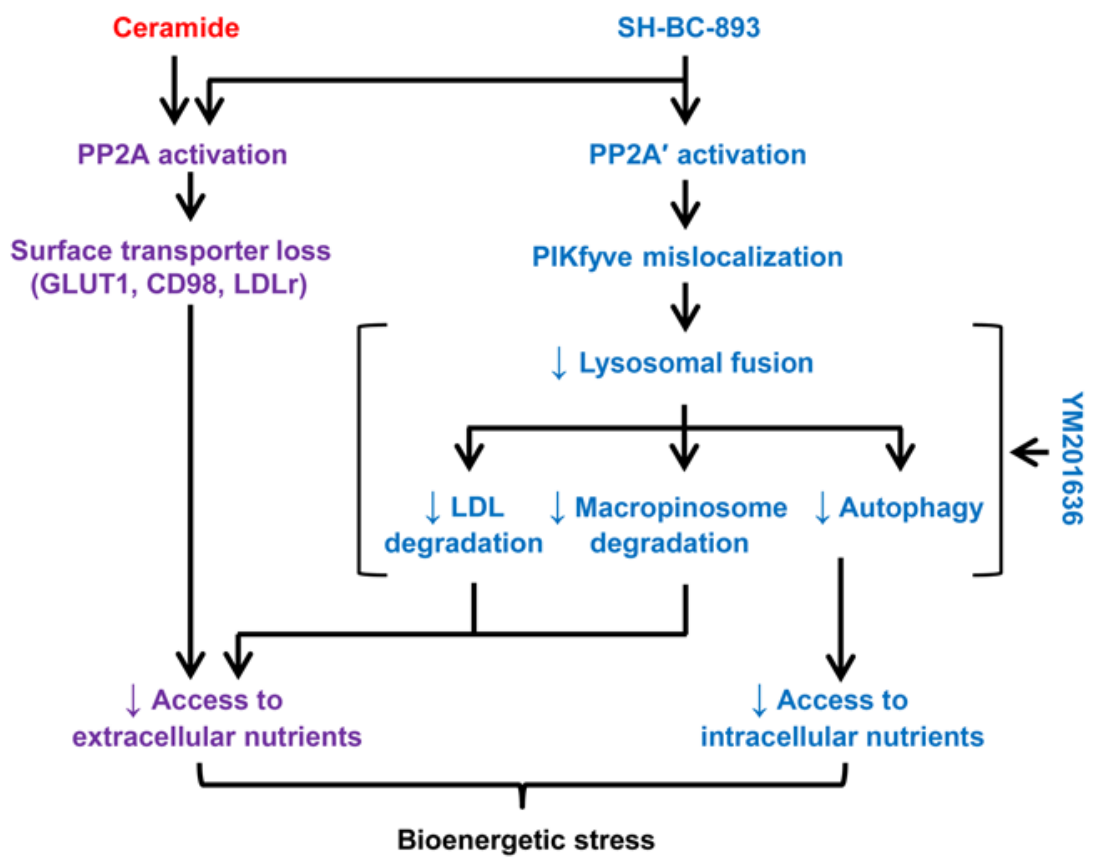

Figure 9. Vacuolating sphingolipid SH-BC-893 targets primary and adaptive pathways for nutrient acquisition. Like ceramide, the synthetic sphingolipid SH-BC-893 activates PP2A to downregulate nutrient transporters. In addition, SH-BC-893 activates a second PP2A complex, PP2A' that is not affected by ceramide. Activation of PP2A' leads to mislocalization of PIKfyve and $\mathrm{PI}(3,5) \mathrm{P}_{2}$, reducing lysosomal fusion reactions. Because $\mathrm{PI}(3,5) \mathrm{P}_{2}$ is membrane anchored and cannot diffuse to its target, loss of $\mathrm{PI}(3,5) \mathrm{P}_{2}$ (YM201636 treatment) and $\mathrm{PI}(3,5) \mathrm{P}_{2}$ mislocalization (SH-BC-893 treatment) produce similar phenotypes. While ceramide limits access to extracellular nutrients, SH-BC-893 blocks access to both extracellular and intracellular nutrients. Substrate limitation in the context of oncogene-driven anabolism is lethal, while nontransformed cells can make adaptive metabolic changes that allow them to survive nutrient stress.

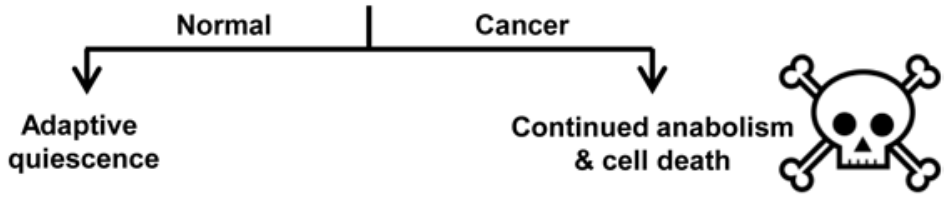

data indicate that $\mathrm{SH}-\mathrm{BC}-893$ blocks autophagic flux at multiple levels through effects on PIKfyve, probably enhancing the bioenergetic stress induced by transporter downregulation (Figure 1).

In cells with activated Ras, macropinocytosis might also confer resistance to nutrient transporter downregulation (9). However, $\mathrm{PI}(3,5) \mathrm{P}_{2}$ is also required for macropinosome degradation (51). While $\mathrm{Kras}^{\mathrm{G} 12 D_{-}}$expressing cells efficiently macropinocytosed dextran in an 5-( $N$-ethyl- $N$-isopropyl) amiloride-sensitive (EIPA-sensitive) manner, both YM201636 and SH-BC-893 dramatically reduced macropinosome fusion with lysosomes (Figure 6, G and $\mathrm{H})$. Macropinosomes that fail to fuse with lysosomes would not supply amino acids (8). Thus, by disrupting PIKfyve localization, SH-BC-893 limited access to lysosomally derived nutrients (Figure 6) at the same time that it downregulated transporters for amino acids and glucose (Figure 1, C and D).

Vacuolation increases the antineoplastic activity of $\mathrm{SH}-\mathrm{BC}-893$. To assess the relative contribution of vacuolation to the antineoplastic activity of SH-BC-893, cells were treated with YM201636 (vacuolation only) and ceramide (transporter loss without vacuolation) alone and in combination. YM201636 was minimally cytotoxic as a single agent but significantly enhanced ceramide-induced death in multiple cancer cell lines, without increasing nutrient transporter loss (Figure 7, A and B, and Supplemental Figure 5D and Supplemental Figure 7A). Moreover, VAC14-overexpressing cells that did not vacuolate (Figure 3F and Figure 6F) were resistant to SH-BC-893- and FTY720-induced death but were not protected from death induced by the nonvacuolating sphingolipid ceramide (Figure 7, C and D, and Supplemental Figure $7 \mathrm{~B})$. Taken together, these data suggest that vacuolation contributes to the ability of SH-BC-893 to kill cancer cells. To assess whether vacuolation enhances the antineoplastic activity of SH-BC-893 in vivo, mice bearing SW480 xenografts expressing empty vector or VAC14 were treated by gavage with vehicle or SH-BC-893. Tumors were harvested while still small in order to limit tumor necrosis that might confound the microscopic analysis of SH-BC-893-induced vacuolation. As seen in vitro, VAC14 overexpression conferred resistance to both vacuolation and growth inhibition by SH-BC-893 (Figure 7, E and F). These results demonstrate that vacuolation contributes to the antineoplastic effects of SH-BC-893 both in vitro and in vivo.

The multifaceted actions of SH-BC-893 confer activity in slower-growing, autochthonous prostate tumors. Because the activity of SH-BC-893 was linked to metabolic rate (Figure 2, A and $\mathrm{B})$, it was not clear whether slower-growing tumors that do not exhibit the classic Warburg phenotype would also be sensitive. To test this, SH-BC-893 was evaluated in a validated genetically engineered mouse model for invasive castration-resistant prostate cancer that lacks p53 and PTEN expression exclusively in the prostate ( $p 53^{-/-}$Pten $^{-/}$mice, hereafter referred to as $\mathrm{pDKO}$ mice) (52-54). Cells derived from tumors in these mice (murine prostate cancer epithelial cells [mPCEs]) exhibited reduced glycolysis and were not dependent on extracellular glucose and amino acids for survival (Figure 8, A and B). However, SH-BC-893 still produced a phenotype consistent with starvation, as the bound $\mathrm{NADH}$ fraction increased and cell-permeant nutrients protected mPCEs from death (Figure 8, A and C). Prostate cancer cells depend on exogenous LDL for growth and survival (5). Interestingly, SH-BC-893 not only vacuolated MPCEs and downregulated $4 \mathrm{~F} 2 \mathrm{HC}$, but also dramatically decreased surface levels of the LDL receptor (LDLr), LDL uptake, and lipid droplet accumula- 
tion (Figure 8, D-H, Supplemental Figure 3B, and Supplemental Figure 8, A and B). Ceramide and YM201636 both reduced surface LDLr levels, but LDLr accumulated in different intracellular compartments (Supplemental Figure 8A). Consistent with their ability to block lysosomal fusion (Figure 6), both YM201636 and SH-BC-893 reduced LDL colocalization with LysoTracker Blue (Figure 8, F and G). In keeping with the inhibition of LDL degradation in lysosomes, cellular lipid droplet content was inversely correlated with the extent of vacuolation (Supplemental Figure $8 \mathrm{~B})$. In summary, $\mathrm{SH}-\mathrm{BC}-893$ starves prostate cancer cells for essential nutrients, both by downregulating transporters and by blocking lysosomal nutrient generation.

We next evaluated the effect of SH-BC-893 on prostate tumor growth in vivo. In C57BL/6 mice bearing $\mathrm{mPCE}$ s.c. isografts, 60 $\mathrm{mg} / \mathrm{kg} \mathrm{SH-BC}-893$ given daily by gavage slowed tumor growth by $60 \%$, similar to the results seen with SW620 xenografts dosed with $20 \mathrm{mg} / \mathrm{kg}$ i.p. (Figure 2, G-I and Figure 8I). At $120 \mathrm{mg} / \mathrm{kg}$, SH-BC-893 inhibited prostate tumor growth by more than $90 \%$ (Figure 8I). Similarly, SH-BC-893 decreased autochthonous tumor growth in pDKO mice by $62 \%(60 \mathrm{mg} / \mathrm{kg})$ or $82 \%(120$ $\mathrm{mg} / \mathrm{kg}$ ) (Figure 8J). Histologically, SH-BC-893 slowed prostate tumor progression, eliminating invasive disease and dramatically reducing cellular pleomorphism, hyperchromasia, and nuclear atypia; SH-BC-893-treated mice exclusively exhibited prostatic intraepithelial neoplasia, whereas adenocarcinoma was present in all vehicle-treated animals (Supplemental Figure 9). As expected, if amino acid transporters were downregulated (Figure 8D), TORC1-dependent ribosomal protein S6 phosphorylation was reduced in SH-BC-893-treated tumors (Supplemental Figure 8C). Akt activity was slightly elevated by SH-BC-893, consistent with a loss of TORC1-mediated negative feedback. Thus, SH-BC-893 is effective against tumors with distinct molecular defects and metabolic characteristics.

SH-BC-893 produces equivalent transporter loss and vacuolation in normal and transformed cells and therefore limits the access of normal cells to nutrients (Figures 1, C and E, and Supplemental Figure 3, B and C). Consistent with this, SH-BC-893treated pDKO mice gained less weight than did control mice (Supplemental Figure 8D). However, even mice treated with the highest dose of SH-BC-893 gained weight, and all treated mice exhibited normal behavior and activity levels. Blood chemistry analysis at sacrifice indicated that $\mathrm{SH}-\mathrm{BC}-893$ was not toxic to the liver or kidneys at the antineoplastic dose (Supplemental Table 1). The slight elevation in serum creatine phosphokinase in animals treated with $120 \mathrm{mg} / \mathrm{kg} \mathrm{SH}-\mathrm{BC}-893$ is suggestive of mild muscle catabolism in response to nutrient restriction. Importantly, proliferating normal tissues were minimally affected by SH-BC-893, as evidenced by normal complete blood counts and histopathology of intestinal crypts in mice treated with $120 \mathrm{mg} / \mathrm{kg} \mathrm{SH-BC-893}$ for 11 weeks (Supplemental Table 2 and Supplemental Figure 8E). The lack of toxicity to normal tissues is consistent with our finding that nontransformed cells can adapt to nutrient stress that triggers a bioenergetic crisis in less metabolically flexible tumor cells (Figure 2, D and E). In conclusion, blocking parallel nutrient access pathways by disrupting membrane trafficking is a safe and effective means to target constitutive anabolism in cancer cells with divergent metabolic programs.

\section{Discussion}

These studies demonstrate that synthetic sphingolipids with vacuolating properties starve multiple classes of cancer cells to death, while sparing normal tissues. SH-BC-893 was effective against tumors with distinct metabolic signatures and growth characteristics, most likely because it inhibits both primary and adaptive nutrient acquisition pathways, downregulating cell surface transporters/receptors, while simultaneously blocking the lysosomal degradation of autophagosomes, macropinosomes, and LDL particles (Figure 9) (17). PP2A, the target of SH-BC-893, is activated in both nontransformed and cancer cells, leading to nutrient transporter loss and profound vacuolation (Figures 1, C and D, Supplemental Figure 2C and Supplemental Figure 3, A-C). However, nontransformed cells survive SH-BC-893-induced nutrient stress due to their ability to make appropriate metabolic adaptations; oncogenic mutations lock cancer cells into a progrowth metabolic program that sensitizes them to nutrient stress (Figure 2, A-F, and Supplemental Figure 2, A and B). By blocking multiple nutrient access pathways, $\mathrm{SH}-\mathrm{BC}-893$ acts as a cellular starvation mimetic; tumor cells are also hypersensitive to dietary restriction and intermittent fasting (55). However, as dietary restriction is ineffective against PTEN-deficient tumors (56) and SH-BC-893 profoundly inhibited the growth of PTEN-null prostate tumors (Figure 8, I and J), cellular and organismal nutrient limitation probably limits tumor growth through distinct mechanisms. While SH-BC-893 was not toxic to normal proliferative tissues at the antineoplastic dose (Figure 2F, Supplemental Figure 8E, Supplemental Table 1 and Supplemental Table 2), a consideration with any cancer therapy that limits nutrient access is how it would affect cachexic patients. Cancer cachexia has a complex etiology and is not simply a consequence of reduced food intake (57). It is possible that compounds that limit tumor lactate production might actually benefit cachexic patients if they reduce the futile Cori cycle through which the liver converts tumor lactate into glucose. How SH-BC-893 administration affects whole-body metabolism and cytokine levels merits further investigation.

As a single agent, $\mathrm{SH}-\mathrm{BC}-893$ acts like a combination therapy by targeting the multiple nutrient acquisition pathways that fuel cancer anabolism (17). It will be important to test whether pairing SH-BC-893 with drugs that target oncogenic signal transduction pathways will increase efficacy. Our results provide a strong rationale for combining SH-BC-893 with PI3 kinase inhibitors (58). Akt activity increased in SH-BC-893-treated prostate tumors (Supplemental Figure 8C), most likely due to the loss of TORC1-dependent negative feedback in cells in which SH-BC-893 restricted access to amino acids. Suppressing Akt activity while administering SH-BC-893 might increase tumor growth inhibition or even cause tumor regression. The combination with androgen-signaling inhibitors might also be valuable; the studies shown in Figure 8, I and J, were performed in intact male mice. Importantly, there are currently no other agents that can reconstitute the multifaceted effects of SH-BC-893. While PIKfyve kinase inhibitors such as apilimod (59) might induce vacuolation, PIKfyve inhibition alone does not reduce surface transporter levels (Supplemental Figure 5D). While Akt activates PIKfyve (60), Akt inhibitors do not induce vacuolation or transporter loss (data not shown and ref. 17). Indeed, no agents other 
than sphingolipids coordinately downregulate transporters for multiple nutrients. Thus, $\mathrm{SH}-\mathrm{BC}-893$ is a unique, apical inhibitor of cancer metabolism that works by blocking parallel, partially redundant nutrient access pathways (17).

The development of resistance limits the effectiveness of targeted therapies. Because sensitivity to SH-BC-893 is linked to an intrinsic property of cancer cells - constitutive anabolism, rather than a particular genetic lesion - it may be more difficult for cancer cells to become resistant to the metabolic effects of SH-BC-893. Indeed, individual oncogenic mutations were sufficient to limit the metabolic flexibility of nutrient-stressed cells and sensitize them to SH-BC-893-induced death (Figure 2, D and E, and Supplemental Figure 2, A and B). The ability of SH-BC-893 to simultaneously block 4 key pathways for nutrient acquisition (glucose and amino acid transporters, LDLr, macropinocytosis, and autophagy) also limits the options for acquired resistance. These multifaceted actions should also reduce the ability of tumor heterogeneity to confer drug resistance, as tumor cells with very different metabolic profiles are equally sensitive (Figure 2 and Figure 8). Although metabolic reprogramming may be unlikely to afford resistance, cancer cells could become insensitive to SH-BC-893 because of mutations in the target or downstream pathways. Eliminating global PP2A activity is cell lethal and therefore not a viable resistance strategy. However, cancer cells may acquire resistance by reducing the activity of the specific PP2A complexes activated by SH-BC-893. Genetic alterations that limit vacuolation may also reduce sensitivity to SH-BC-893. A cBioPortal search (http://www.cbioportal.org/) revealed that VAC14, a protein that confers partial resistance to SH-BC-893 (Figure 7, C-F), is amplified in $27 \%$ of breast cancer xenografts in one dataset, but amplification was not detected in primary patient breast tumors. On the other hand, VAC14 is deleted in $3 \%$ to $6 \%$ of prostate tumors, which might conversely sensitize these cancers to SH-BC-893. It is interesting that we were able to generate SW480 but not SW620 cells that stably overexpressed VAC14. SW620 and SW480 were isolated from the same patient, but SW480 cells were derived from the primary tumor site and SW620 from a lymph node metastasis. It is possible that VAC14 overexpression is poorly compatible with mutations that confer tumor aggressiveness and metastatic potential. Identifying biomarkers that correlate with sensitivity and resistance to SH-BC-893 will help to clarify this issue.

In conclusion, water-soluble and orally bioavailable vacuolating sphingolipid drugs like SH-BC-893 could provide a cell biology-rather than a biochemistry-based approach to targeting "cancer metabolism." Although optimization of pharmacological properties, formulation, dose, and schedule of administration is warranted, the water solubility, oral activity, micromolar trough levels, and accumulation in tumors suggest that SH-BC-893 has good druglike properties and is worthy of further preclinical evaluation.

\section{Methods}

Cell culture and reagents. OP9, DLD-1, SW48, SW620, MDA-MB-231, PANC-1, HOS, MG-63, and Zr75-1 cells were obtained from the ATCC. SW480 cells were provided by Marian Waterman (UCI); HeLa cells by Christine Sütterlin (UCI); LS180 cells by Bruce Blumberg (UCI); and FL5.12 cells by Craig Thompson (Memorial Sloan Kettering Cancer
Center, New York, New York, USA). p53-/- LSL-Kras ${ }^{G 12 D}$ MEFs with and without Cre-mediated deletion of the STOP cassette were provided by David Tuveson (Cold Spring Harbor Laboratory, Cold Spring Harbor New York, USA). Primary $p 53^{+/+} \mathrm{Pten}^{+/+}, p 53^{-/-} \mathrm{Pten}^{+/+}$, and $p 53^{-/-} \mathrm{Pten}^{-}$ - MEFs were generated in-house from embryos from C57BL/6 mice using standard techniques. mPCE cells generated from $p 53^{-/-} \mathrm{Pten}^{-/-}$ mouse prostate tissue were maintained in DMEM with 10\% FBS, 25 $\mu \mathrm{g} / \mathrm{ml}$ bovine pituitary extract, $5 \mu \mathrm{g} / \mathrm{ml}$ bovine insulin, and $6 \mathrm{ng} /$ $\mathrm{ml}$ recombinant human EGF. FL5.12 cells were maintained in RPMI 1640 medium supplemented with 10\% FCS, $10 \mathrm{mM}$ HEPES, $55 \mu \mathrm{M}$ 2-mercaptoethanol, antibiotics, $2 \mathrm{mM} \mathrm{L-glutamine,} \mathrm{and} 500 \mathrm{pg} / \mathrm{ml}$ rIL-3. FL5.12 cells were adapted to grow in $25 \mathrm{pg} / \mathrm{ml} \mathrm{IL-3} \mathrm{by} \mathrm{gradually}$ reducing the IL-3 concentration over 2 weeks of culture. DLD-1 and Zr75-1 cells were cultured in the same media as FL5.12, but without IL-3. HeLa, OP9, MG-63, and MEF cells were cultured in DMEM with $4.5 \mathrm{~g} / 1$ glucose and L-glutamine supplemented with $10 \%$ FCS and antibiotics. Starvation medium was produced by making DMEM lacking amino acids and glucose from chemical components and supplementing with $10 \%$ dialyzed FCS. LS180, SW48, PANC-1, MDAMB-231, SW480, and SW620 cells were cultured in DMEM supplemented with $10 \%$ FCS, antibiotics, and $1 \mathrm{mM}$ sodium pyruvate. Cell viability was measured by vital dye exclusion using either propidium iodide or DAPI at $1 \mu \mathrm{g} / \mathrm{ml}$. Analysis of cell-surface $4 \mathrm{~F} 2 \mathrm{HC}$ levels with PE-conjugated anti-CD98 was restricted to viable cells as determined by DAPI exclusion. Anchorage-independent growth of SW620 cells was measured in DMEM-10 containing 0.35\% low-melt agarose with a $0.5 \%$ agarose bottom layer. PBMCs were obtained from the normal blood donor program run by the Clinical and Translational Science Award (CTSA)-supported Institute for Clinical and Translational Sciences (ICTS) at UCI. P4OPx-EGFP plasmid was provided by Seth Field (UCSD, San Diego, California, USA), mCherry-VAC14 ${ }^{\mathrm{WT}}$ and mCherry-VAC14 ${ }^{\mathrm{L} 156 \mathrm{R}}$ plasmids by Thomas Weide (University Hospital of Muenster, Germany), mCherry-TRPML1, EGFP-TRPML1, and mCherry-ML1N ${ }^{\star} 2$ plasmids by Haoxing Xu (University of Michigan), and PIKfyve and VAC14 shRNA by Anand Ganesan (UCI).

Light microscopy. Bright-field and epifluorescence microscopy were conducted using a Nikon TE2000-S fluorescence microscope; confocal microscopy was performed on a Zeiss LSM780 confocal microscope or a Nikon Eclipse Ti spinning-disk confocal microscope. The following antibodies were used: murine 4F2HC (catalog 128208; eBioscience) and LAMP1 (catalog 53-1079-42; eBioscience); human 4F2HC (catalog 556077; BD Biosciences); human GLUT1 (catalog NB300-666; Novus Biologicals); LC3 (catalog 4108; Cell Signaling Technology); PIKfyve (catalog 4082; Tocris); VAC14 (catalog SAB4200074; Sigma-Aldrich); and WIPI2 (catalog LS-C154557100; LifeSpan Biosciences). Colocalization was determined using the JACoP plugin in ImageJ software (NIH). Macropinocytosis was measured after a 16-hour incubation in serum-free DMEM. $p 53^{-/-}$ LSL-Kras ${ }^{G 12 D}$ MEFs before ( $L S L$ or after ( ras $^{G 12 D}$ ) introduction of Cre were serum starved for 16 hours and then treated with the macropinocytosis inhibitor 5-( $N$-ethyl- $N$-isopropyl) amiloride (EIPA) (75 $\mu \mathrm{M}$ ) for 1 hour or SH-BC-893 for 6 hours. Oregon Green dextran (Life Technologies, Thermo Fisher Scientific; catalog D7173) $(1 \mathrm{mg} / \mathrm{ml})$ and LysoTracker Red (Life Technologies, Thermo Fisher Scientific; catalog L-7525) (1:2,000 dilution) were added for 30 minutes, and live cells were evaluated on the spinning-disc confocal microscope. For Dil-LDL uptake, mPCEs were incubated in media with $10 \%$ char- 
coal-stripped serum for 24 hours, then incubated with $20 \mu \mathrm{g} / \mathrm{ml}$ DilLDL (Life Technologies, Thermo Fisher Scientific; catalog L3482), with or without SH-BC-893, for 6 hours and LysoTracker Blue (Life Technologies, Thermo Fisher Scientific; catalog L-7525), for $30 \mathrm{~min}-$ utes. To detect surface LDL receptors, Dil-LDL was added at $4^{\circ} \mathrm{C}$ to mPCEs treated with SH-BC-893 for 3 hours, or cells were stained with LDL receptor antibody (R\&D Systems; catalog AF2255). SW480 tumors were excised and fixed in $2.5 \%$ glutaraldehyde in $0.1 \mathrm{M}$ phosphate buffer ( $\mathrm{pH} 7.4)$ and stored in the dark at $4^{\circ} \mathrm{C}$ until embedment. Tumor samples were processed by the Pathology Research Services Core Facility at UCI.

Electron microscopy. FL5.12 cells treated with FTY720 were fixed with $2.5 \%$ glutaraldehyde and $2.5 \%$ formaldehyde in $0.1 \mathrm{M}$ sodium cacodylate buffer and stored at $4^{\circ} \mathrm{C}$ until embedment. Cells were postfixed with $1 \%$ osmium tetroxide, serially dehydrated, and embedded in eponat12 resin. Ultra-thin sections were cut, mounted on grids, and stained with uranyl acetate and lead citrate. Samples were analyzed on a Philips CM10 transmission electron microscope. Representative images are shown from 2 independent experiments.

In vivo studies. Experiments were conducted in mice following a power analysis conducted in consultation with the Biostatistics Shared Resource of the Chao Family Comprehensive Cancer Center at UCI. Xenografts were produced by injecting 5 million cells s.c. into the flank of 10- to 16-week-old male or female NSG mice. Prostate isografts were produced in the same manner, but in male 6- to 8-week-old C57BL/6 mice. Once tumors reached $100 \mathrm{~mm}^{3}$, SH-BC-893 was administered by i.p. injection (Figure 2, G-I) or oral gavage (Figure 7, E and F, and Figure 8, I and J) as indicated. Tumor volume was calculated using the following formula: volume $\left(\mathrm{mm}^{3}\right)$ $=$ length $[\mathrm{mm}] \times(\text { width }[\mathrm{mm}])^{2} \times 0.52$; bioluminescence was measured using an in vivo imaging system (IVIS) (Xenogen). To generate pDKO mice on a C57BL6 background, Pten ${ }^{\text {flox }}$ mice (stock no. 0045597) and $p 53^{\text {flox }}$ mice (stock no. 008462) were obtained from The Jackson Laboratory, and $\mathrm{PB}$-Cre 4 mice (strain no. 01XF5) were obtained from the National Cancer Institute's Frederick Mouse Repository. Age-matched cohorts of pDKO males were generated by in vitro fertilization, executed with the assistance of the Transgenic Mouse Facility at UCI. Treatment was begun at 6 to 7 weeks of age. Tumor weight was determined by isolating the complete genitourinary (GU) tract of pDKO mice and subtracting the average weight of a normal GU tract from that of age-matched mice $(n=3)$ after it was determined that SH-BC-893 treatment of normal mice did not alter GU tract weight $(n=3)$. Tumor samples were processed and imaged by the Pathology Research Services Core Facility at UCI. Blood chemistry was analyzed by IDEXX BioResearch, and complete blood counts were performed using a Hemavet hematology system (Drew Scientific Group, Erba Diagnostics).

$N A D H$ fluorescence lifetime imaging microscopy. Lifetime images were acquired using a Zeiss 780 microscope coupled to a Mai Tai Ti:Sapphire laser system (Spectra-Physics). The excitation wavelength was $740 \mathrm{~nm}$, and a dichroic filter $(690 \mathrm{~nm})$ was used to separate the fluorescence signal from the laser light. A $63 \times 1.15$ water-immersion objective was used. The image acquisition settings were: image size of $256 \times 256$ pixels and scan speed of $25.21 \mu \mathrm{sec} /$ pixel. Fluorescence was detected by a hybrid detector (HPM-100; Hamamatsu). Data were collected until 100 counts in the brightest pixel of the image were acquired. The fluorescence lifetime imaging microscopy (FLIM) sys- tem was calibrated during each imaging session by measuring the fluorescence decay of fluorescein with a single exponential of $4.04 \mathrm{nsec}$. Phasor transformation of FLIM images and analysis of the average lifetime in single cells were done as described previously $(30,35,36)$. Data were processed using SimFCS software developed at the Laboratory of Fluorescence Dynamics at UCI. The nucleus was excluded when determining the bound NADH fraction. The mean \pm SEM is shown; $n \geq 45$ cells from 2 independent experiments (Figure $1 \mathrm{~F}$ and Figure 2, B and D).

Coherent anti-Stokes Raman spectroscopy. The coherent anti-Stokes Raman spectroscopy (CARS) imaging system is described in detail in ref. 61 . Cells were fixed with $4 \%$ formaldehyde and imaged with a $60 \times$ water objective. The laser power on the sample was at $10 \mathrm{~mW}$, with a 10-ms pixel dwell time. The lipid droplet area was estimated from CARS images using a customized MATLAB (MathWorks) program. Four components, the Otsu thresholding method was used to separate the lipid droplets, cell cytoplasm, cell nucleus, and the background. The lipid droplet area was defined as the number of pixels covered by lipid droplets over the number of pixels covered by cytoplasm.

PIKfyve in vitro kinase assay. FLAG-PIKfyve was expressed in HEK293T cells, purified with FLAG-beads, and eluted with FLAG peptides. PI3P and phosphatidylserine (C16) liposomes were generated by sonication in $2 \times$ lipid mixture buffer (40 mM Tris-HCl, pH 7.4, $200 \mathrm{mM}$ $\mathrm{NaCl}, 1 \mathrm{mM}$ EGTA), with or without FTY720. FLAG-PIKfyve and lipid mixtures were incubated with $\mathrm{Mg}^{2+}$-ATP solution (6.5 mM HEPES, $\mathrm{pH}$ 7.3, $2.5 \mathrm{mM} \mathrm{MnCl}_{2}, 10 \mathrm{mM} \mathrm{MgCl}, 1 \mathrm{mM} \beta$-glycerophosphate, 0.1 $\mathrm{mM}$ ATP and $\left.\left[{ }^{32} \mathrm{P}\right]-\gamma-\mathrm{ATP}\right)$ for 15 minutes at room temperature. The reaction was stopped by adding $4 \mathrm{M} \mathrm{HCl}$, and phosphoinositides were extracted with methanol/chloroform (1:1). Phosphoinositides were spotted on silica thin-layer chromatographic plates and separated with $2 \mathrm{M}$ acetic acid/1-propanol (35:65). Membranes were dried, exposed to a Phospho Imager, and the counts from $\mathrm{PI}(3,5) \mathrm{P}_{2}$ spots quantified with ImageQuant (GE Healthcare Lifesciences).

Measurement of PI $(3,5) P_{2}$ by HPLC. HeLa cells were rinsed twice with PBS and incubated for 48 hours in inositol labeling medium (inositol-free DMEM containing $5 \mu \mathrm{g} / \mathrm{ml}$ transferrin, $5 \mu \mathrm{g} / \mathrm{ml}$ insulin, $10 \%$ dialyzed FCS, $20 \mathrm{mM}$ HEPES, $2 \mathrm{mM}$ L-glutamine) and $10 \mu \mathrm{Ci} / \mathrm{ml}$ myo$\left[2-{ }^{3} \mathrm{H}\right]$-inositol. Cells were lysed with $4.5 \%$ perchloric acid, scraped, and centrifuged at $14,000 \times g$ for 10 minutes at $4^{\circ} \mathrm{C}$. Cell pellets were rinsed with $100 \mathrm{mM}$ EDTA, centrifuged again, and resuspended in $50 \mu \mathrm{l}$ water. To deacylate lipids, $1 \mathrm{ml}$ methanol, $40 \%$ methylamine, and butanol (45.7\% methanol, $10.7 \%$ methylamine, $11.4 \%$ butanol) was added, and then the mixture was transferred to a glass vial and incubated at $55^{\circ} \mathrm{C}$ for 1 hour. After cooling to room temperature, samples were vacuum dried and resuspended in $0.5 \mathrm{ml}$ water. Lipids were extracted twice with an equal volume of butanol/ethyl ether/ethyl formate (20:4:1). The aqueous phase was vacuum dried and resuspended in $75 \mu \mathrm{l}$ water, and $50 \mu \mathrm{l}$ of each sample was analyzed by HPLC. PI $(3,5)$ $\mathrm{P}_{2}$ levels were expressed as a percentage of total phosphatidylinositol.

PP2A phosphatase activity. PP2A activity was measured using a PP2A immunoprecipitation phosphatase assay kit (EMD Millipore). Briefly, the catalytic subunit of PP2A was immunoprecipitated from FL5.12 cell lysates with $4 \mu \mathrm{g}$ anti-PP2A, C subunit. After 4 washes, the activity of immunoprecipitated PP2A was assessed by dephosphorylation of the phosphopeptide according to the manufacturer's instructions in the presence or absence of $\mathrm{C}_{2}$-ceramide, dihydro- $\mathrm{C}_{2}$-ceramide, FTY720, SH-BC-893, or calyculin A. 
Massspectrometric quantification of $S H-B C-893$. As an internal standard, $75 \mathrm{ng}$ FTY720 was added to $50 \mu \mathrm{l}$ plasma or $50 \mu \mathrm{l}$ tumor homogenate (0.25 M sucrose, $25 \mathrm{mM} \mathrm{KCl}, 50 \mathrm{mM}$ Tris $\mathrm{HCl}, 0.5 \mathrm{mM}$ EDTA, pH 7.4; $1: 9$ weight/volume) combined 1:1 with acetonitrile. Proteins were precipitated and removed by centrifugation (10 min at 18,000 $\mathrm{g}$ ) and the supernatant transferred to a fresh tube on ice containing $50 \mu \mathrm{l}$ acetonitrile plus $0.2 \%$ acetic acid. After a second deproteination with acetonitrile plus $0.2 \%$ acetic acid, $20 \mu$ of the deproteinated samples were analyzed by ultra-performance liquid chromatography tandem mass spectrometry (UPLC-MS/MS) using a Waters Micromass Quattro Premier XE equipped with a C18 reversed-phase column (Waters) with an acetonitrile plus $0.2 \%$ acetic acid gradient elution. The instrument was operated in positive ion mode. Ion transition channels for multiple reaction monitoring were $290 \rightarrow 104$ for SH-BC-893 with a dwell time of $200 \mathrm{msec}$. The cone voltage was $30 \mathrm{~V}$. Standard curves used for quantitation were linear from $50-1,000 \mathrm{ng} / \mathrm{ml}$, with an $R^{2}$ of 0.98 or greater. Recovery of the internal standard was $80 \%$ or more. Tumor concentrations were calculated assuming that $1 \mathrm{~g}=1 \mathrm{ml}$.

Statistics. Significance was determined using a 2-tailed paired $t$ test for single pairwise comparisons. Tukey's method was used, and adjusted $P$ values are reported where multiple comparisons were made. In tumor studies for which data were not normally distributed, a Mann-Whitney $U$ test was used to compare treated mice with control mice. For lipid droplet area in CARS experiments, the mean values between the control and experimental groups were compared with a 2-tailed ANOVA. A $P$ value of less than 0.05 was considered statistically significant.

Study approval. Experiments conducted in mice were approved by and performed in accordance with the IACUC of UCI. PBMCs were obtained from the normal blood donor program run by the CTSA-supported Institute for Clinical and Translational Science at UCI under IRB protocols 2015-1883 (A.L. Edinger) and 2001-2058 (ICTS).

\section{Author contributions}

ALE, SMK, and SH conceptualized the overall project. SMK carried out all experiments except those depicted in Figure $1 \mathrm{~B}$ and Figure 2, A and F (ANM); Figure 1C (MUR); Figure 1D and Figure 8D (BTF); Supplemental Figure 2B and parts of Figure 2C (AR); Figure 2, G-I, and Supplemental Figure 2, D and F (RJM); Figure 3B and Supplemental Figure 3E (ALE); Figure 3, C and D, and Supplemental Figure 3D (GGG); Figure 4A (SK); Figure 4B and Supplemental Figure 6A (YZ); parts of Figure 7, B-F, Supplemental Figure 5, D and E, and Supplemental Figure 7, A and B (TMN); Figure 8, B and C (TTN); Figure $8 \mathrm{H}$ and Supplemental Figure 8B (JH); Figure
8, I and J, Supplemental Figure 8D, part of Supplemental Figure 8, C and E, Supplemental Figure 9, and Supplemental Tables 1 and 2 (SGR); Supplemental Figure 2E (ES); Supplemental Figure $5 \mathrm{~A}$ (TW); and Supplemental Figure 8E and Supplemental Figure 9 (RAE). The methods were developed by SMK, SGR, BC, RJM, ANM, YZ, SK, JH, ES, MK, ATS, LSW, EOP, BJT, SH, and ALE. ALE and SMK wrote the manuscript and prepared the figures. All authors reviewed and edited the text and figures. Resources were provided by BC (SH-BC-893) and MK (S1P reporter MEFs). ATS, LSW, EOP, BJT, SH, and ALE supervised the research. The project was administered by ALE. Funding for the work was provided by ATS, LSW, EOP, BJT, SH, and ALE.

\section{Acknowledgments}

The authors thank Jennifer Prescher, Susanne Rafelski, Michelle Digman, Adeela Syed, Kris Kenney, Richard Van Etten, Jon Neumann, Farah Aktar (UC Irvine, Irvine, California, USA) and Richard Proia (NIH, Bethesda, Maryland, USA) for advice, reagents, and/or access to equipment; Seth Field, Thomas Weide, Ana Maria Cuervo (Albert Einstein College of Medicine, Bronx, New York, USA), Haoxing $\mathrm{Xu}$, and Anand Ganesan for plasmids; and Marian Waterman and David Fruman (UC Irvine, Irvine, California, USA) for comments on the manuscript. This work was supported by grants to ALE from the NIH (R01 GM089919, R21 CA178230); the Congressionally Directed Medical Research Programs (CDMRP) (W81XWH-11-1-0535 and W81XWH-15-1-0010); the American Cancer Society (RSG-11-111-01-CDD); the William Lawrence and Blanche Hughes Foundation; and the UCI Council on Research Computing and Libraries (CORCL). Support was also provided by NIH grants to LSW (R01 NS064015 and R01 GM050403) and ATS (R01 NS089815). BJT, EOP, and JH were supported by Laser Microbeam and Medical Program (LAMMP) grant P41EB015890 from the NIH, National Institute of Biomedical Imaging and Bioengineering (NIBIB). SMK was supported by Graduate Assistance in Areas of National Need (GAANN) grant P200A120207 and ES by NIH grant T32 CA009054. The core facilities at UCI were supported by Cancer Center Support grant P30 CA62203 and the normal blood donor program by National Center for Advancing Translational Sciences (NCATS) grant UL1 TR000153.

Address correspondence to: Aimee Edinger, 2128 Natural Sciences 1, University of California Irvine, Irvine, California 92697, USA. Phone: 949.824.1921; E-mail: aedinger@uci.edu.
1. McCracken AN, Edinger AL. Nutrient transporters: the Achilles' heel of anabolism. Trends Endocrinol Metab. 2013;24(4):200-208.

2. Garcia-Cao I, et al. Systemic elevation of PTEN induces a tumor-suppressive metabolic state. Cell. 2012;149(1):49-62.

3. Shroff EH, et al. MYC oncogene overexpression drives renal cell carcinoma in a mouse model through glutamine metabolism. Proc Natl Acad Sci U S A. 2015;112(21):6539-6544.

4. Ying H, et al. Oncogenic Kras maintains pancreatic tumors through regulation of anabolic glucose metabolism. Cell. 2012;149(3):656-670.

5. Yue S, et al. Cholesteryl ester accumulation induced by PTEN loss and PI3K/AKT activation underlies human prostate cancer aggressiveness. Cell Metab. 2014;19(3):393-406.

6. Guillaumond F, et al. Cholesterol uptake disruption, in association with chemotherapy, is a promising combined metabolic therapy for pancreatic adenocarcinoma. Proc Natl Acad Sci U S A. 2015;112(8):2473-2478.

7. Edinger AL, Thompson CB. Akt maintains cell size and survival by increasing mTOR-dependent nutrient uptake. Mol Biol Cell. 2002;13(7):2276-2288.

8. Palm W, Park Y, Wright K, Pavlova NN, Tuveson DA, Thompson CB. The Utilization of Extracellular Proteins as Nutrients Is Suppressed by mTORC1. Cell. 2015;162(2):259-270.

9. Commisso C, et al. Macropinocytosis of protein is an amino acid supply route in Ras-transformed cells. Nature. 2013;497(7451):633-637.

10. Pieters R, et al. L-asparaginase treatment in acute lymphoblastic leukemia: a focus on Erwinia asparaginase. Cancer. 2011;117(2):238-249.

11. Feun LG, Kuo MT, Savaraj N. Arginine deprivation in cancer therapy. Curr Opin Clin Nutr Metab Care. 2015;18(1):78-82.

12. Jain M, et al. Metabolite profiling identifies a key role for glycine in rapid cancer cell proliferation. Science. 2012;336(6084):1040-1044.

13. Maddocks OD, et al. Serine starvation induces stress and p53-dependent metabolic remodelling in cancer cells. Nature. 2013;493(7433):542-546.

14. McGranahan N, Swanton C. Biological and ther- 
apeutic impact of intratumor heterogeneity in cancer evolution. Cancer Cell. 2015;27(1):15-26.

15. Patel AP, et al. Single-cell RNA-seq highlights intratumoral heterogeneity in primary glioblastoma. Science. 2014;344(6190):1396-1401.

16. Garraway LA, Jänne PA. Circumventing cancer drug resistance in the era of personalized medicine. Cancer Discov. 2012;2(3):214-226.

17. Selwan EM, Finicle BT, Kim SM, Edinger AL. Attacking the supply wagons to starve cancer cells to death. FEBS Lett. 2016;590(7):885-907.

18. Guenther GG, Peralta ER, Rosales KR, Wong SY, Siskind LJ, Edinger AL. Ceramide starves cells to death by downregulating nutrient transporter proteins. Proc Natl Acad Sci U S A. 2008;105(45):17402-17407.

19. Welsch CA, Roth LW, Goetschy JF, Movva NR. Genetic, biochemical, and transcriptional responses of Saccharomyces cerevisiae to the novel immunomodulator FTY720 largely mimic those of the natural sphingolipid phytosphingosine. J Biol Chem. 2004;279(35):36720-36731.

20. Romero Rosales K, et al. Sphingolipid-based drugs selectively kill cancer cells by down-regulating nutrient transporter proteins. Biochem $\mathrm{J}$. 2011;439(2):299-311.

21. Edinger AL. Starvation in the midst of plenty: making sense of ceramide-induced autophagy by analysing nutrient transporter expression. Biochem Soc Trans. 2009;37(Pt 1):253-258.

22. Camm J, Hla T, Bakshi R, Brinkmann V. Cardiac and vascular effects of fingolimod: mechanistic basis and clinical implications. Am Heart J . 2014;168(5):632-644.

23. White E. Exploiting the bad eating habits of Ras-driven cancers. Genes Dev. 2013;27(19):2065-2071.

24. Neviani P, et al. FTY720, a new alternative for treating blast crisis chronic myelogenous leukemia and Philadelphia chromosome-positive acute lymphocytic leukemia. JClin Invest. 2007;117(9):2408-2421.

25. Azuma H, Takahara S, Horie S, Muto S, Otsuki $\mathrm{Y}$, Katsuoka Y. Induction of apoptosis in human bladder cancer cells in vitro and in vivo caused by FTY720 treatment. JUrol. 2003;169(6):2372-2377.

26. Azuma H, et al. Marked prevention of tumor growth and metastasis by a novel immunosuppressive agent, FTY720, in mouse breast cancer models. Cancer Res. 2002;62(5):1410-1419.

27. Chen B, et al. Azacyclic FTY720 Analogues That Limit Nutrient Transporter Expression but Lack S1P Receptor Activity and Negative Chronotropic Effects Offer a Novel and Effective Strategy to Kill Cancer Cells in Vivo. ACS Chem Biol. 2016;11(2):409-414.

28. Kono M, Tucker AE, Tran J, Bergner JB, Turner EM, Proia RL. Sphingosine-1-phosphate receptor 1 reporter mice reveal receptor activation sites in vivo. J Clin Invest. 2014;124(5):2076-2086.

29. Birsoy K, et al. Metabolic determinants of cancer cell sensitivity to glucose limitation and biguanides. Nature. 2014;508(7494):108-112.

30. Digman MA, Caiolfa VR, Zamai M, Gratton E. The phasor approach to fluorescence lifetime imaging analysis. Biophys J. 2008;94(2):L14-L16.

31. Bird DK, et al. Metabolic mapping of MCF10A human breast cells via multiphoton fluorescence lifetime imaging of the coenzyme NADH. Cancer Res. 2005;65(19):8766-8773.

32. Stringari C, Cinquin A, Cinquin O, Digman MA, Donovan PJ, Gratton E. Phasor approach to fluorescence lifetime microscopy distinguishes different metabolic states of germ cells in a live tissue. Proc Natl Acad Sci U S A. 2011;108(33):13582-13587.

33. Stringari $C$, et al. In vivo single-cell detection of metabolic oscillations in stem cells. Cell Rep. 2015;10(1):1-7.

34. Quesada E, Acuña AU, Amat-Guerri F. New transmembrane polyene bolaamphiphiles as fluorescent probes in lipid bilayers. Angew Chem Int Ed Engl. 2001;40(11):2095-2097.

35. Pate KT, et al. Wnt signaling directs a metabolic program of glycolysis and angiogenesis in colon cancer. EMBO J. 2014;33(13):1454-1473.

36. Stringari C, Edwards RA, Pate KT, Waterman ML, Donovan PJ, Gratton E. Metabolic trajectory of cellular differentiation in small intestine by Phasor Fluorescence Lifetime Microscopy of NADH. Sci Rep. 2012;2:568.

37. Bauer DE, et al. Cytokine stimulation of aerobic glycolysis in hematopoietic cells exceeds proliferative demand. FASEB J. 2004;18(11):1303-1305.

38. Gault CR, Eblen ST, Neumann CA, Hannun YA, Obeid LM. Oncogenic K-Ras regulates bioactive sphingolipids in a sphingosine kinase 1-dependent manner. J Biol Chem. 2012;287(38):31794-31803.

39. McCartney AJ, Zhang Y, Weisman LS. Phosphatidylinositol 3,5-bisphosphate: low abundance, high significance. Bioessays. 2014;36(1):52-64.

40. Jefferies HB, et al. A selective PIKfyve inhibitor blocks PtdIns $(3,5) \mathrm{P}(2)$ production and disrupts endomembrane transport and retroviral budding. EMBO Rep. 2008;9(2):164-170.

41. Rutherford AC, et al. The mammalian phosphatidylinositol 3-phosphate 5-kinase (PIKfyve) regulates endosome-to-TGN retrograde transport. JCell Sci. 2006;119(Pt 19):3944-3957.

42. Zhang Y, et al. Modulation of synaptic function by VAC14, a protein that regulates the phosphoinositides $\mathrm{PI}(3,5) \mathrm{P}_{2}$ and PI(5)P. EMBO J. 2012;31(16):3442-3456.

43. Dong XP, et al. PI $(3,5) \mathrm{P}(2)$ controls membrane trafficking by direct activation of mucolipin $\mathrm{Ca}(2+)$ release channels in the endolysosome. Nat Commun. 2010;1:38.

44. Li X, et al. Genetically encoded fluorescent probe to visualize intracellular phosphatidylinositol 3,5-bisphosphate localization and dynamics. Proc Natl Acad Sci U S A . 2013;110(52):21165-21170.

45. Chalfant CE, Kishikawa K, Mumby MC, Kamibayashi C, Bielawska A, Hannun YA. Long chain ceramides activate protein phosphatase- 1 and protein phosphatase-2A. Activation is stereospe cific and regulated by phosphatidic acid. J Biol Chem. 1999;274(29):20313-20317.

46. Chalfant CE, Szulc Z, Roddy P, Bielawska A, Hannun YA. The structural requirements for ceramide activation of serine-threonine protein phosphatases. JLipid Res. 2004;45(3):496-506.

47. Ogretmen B, Hannun YA. Biologically active sphingolipids in cancer pathogenesis and treatment. Nat Rev Cancer. 2004;4(8):604-616.

48. Wang $\mathrm{W}$, et al. Up-regulation of lysosomal TRPML1 channels is essential for lysosomal adaptation to nutrient starvation. Proc Natl Acad Sci U S A. 2015;112(11):E1373-E1381.

49. Vicinanza M, et al. PI(5)P regulates autophagosome biogenesis. Mol Cell. 2015;57(2):219-234.

50. Zolov SN, et al. In vivo, Pikfyve generates PI $(3,5)$ $\mathrm{P} 2$, which serves as both a signaling lipid and the major precursor for PI5P. Proc Natl Acad Sci U S A. 2012;109(43):17472-17477.

51. Kerr MC, et al. Inhibition of the PtdIns(5) kinase PIKfyve disrupts intracellular replication of Salmonella. EMBO J. 2010;29(8):1331-1347.

52. Wu X, Daniels G, Lee P, Monaco ME. Lipid metabolism in prostate cancer. Am JClin Exp Urol. 2014;2(2):111-120.

53. Schwarzenböck S, Souvatzoglou M, Krause BJ. Choline PET and PET/CT in Primary Diagnosis and Staging of Prostate Cancer. Theranostics. 2012;2(3):318-330.

54. Chen Z, et al. Crucial role of p53-dependent cellular senescence in suppression of Pten-deficient tumorigenesis. Nature. 2005;436(7051):725-730.

55. Lee $\mathrm{C}$, et al. Fasting cycles retard growth of tumors and sensitize a range of cancer cell types to chemotherapy. Sci Transl Med. 2012;4(124):124ra27.

56. Kalaany NY, Sabatini DM. Tumours with PI3K activation are resistant to dietary restriction. Nature. 2009;458(7239):725-731.

57. Argilés JM, Busquets S, Stemmler B, LópezSoriano FJ. Cancer cachexia: understanding the molecular basis. Nat Rev Cancer. 2014;14(11):754-762.

58. Schwartz S, et al. Feedback suppression of PI3Ko signaling in PTEN-mutated tumors is relieved by selective inhibition of PI3K $\beta$. Cancer Cell. 2015;27(1):109-122.

59. Cai X, et al. PIKfyve, a class III PI kinase, is the target of the small molecular IL-12/IL-23 inhibitor apilimod and a player in Toll-like receptor signaling. Chem Biol. 2013;20(7):912-921.

60. Er EE, Mendoza MC, Mackey AM, Rameh LE, Blenis J. AKT facilitates EGFR trafficking and degradation by phosphorylating and activating PIKfyve. Sci Signal. 2013;6(279):ra45.

61. Suhalim JL, et al. Characterization of cholesterol crystals in atherosclerotic plaques using stimulated Raman scattering and second-harmonic generation microscopy. Biophys J. 2012;102(8):1988-1995. 